\title{
Mathematical framework for simulation of quantum fields in complex interferometers using the two-photon formalism
}

\author{
Thomas Corbitt, ${ }^{1}$ Yanbei Chen, ${ }^{2,3}$ and Nergis Mavalvala ${ }^{1}$ \\ ${ }^{1}$ LIGO Laboratory, Massachusetts Institute of Technology, Cambridge, MA 02139 \\ ${ }^{2}$ Theoretical Astrophysics, California Institute of Technology, Pasadena, CA 91125 \\ ${ }^{3}$ Max-Planck-Institut für Gravitationsphysik, Am Mühlenberg 1, 14476 Golm, Germany
}

\begin{abstract}
We present a mathematical framework for simulation of optical fields in complex gravitational-wave interferometers. The simulation framework uses the two-photon formalism for optical fields and includes radiation pressure effects, an important addition required for simulating signal and noise fields in next-generation interferometers with high circulating power. We present a comparison of results from the simulation with analytical calculation and show that accurate agreement is achieved.
\end{abstract}

\section{INTRODUCTION}

Next-generation gravitational-wave (GW) interferometers, such as those planned for Advanced LIGO [1], are designed to have a fifteen-fold improvement in sensitivity over present-day detectors 2]. Among the techniques planned to achieve this improved sensitivity is an increase in the input laser power. The higher laser power reduces the shot noise limit at frequencies above $\sim 100 \mathrm{~Hz}$, as intended, but has the deleterious effect of increasing the radiation-pressure noise at lower frequencies. Consequently, advanced detector sensitivity at almost all frequencies in the detection band is expected to be limited by quantum noise. Qualitatively speaking, shot noise and radiation-pressure noise correspond to measurement noise and back action noise in quantum measurement theory - together they often impose the Standard Quantum Limit (SQL) to measurement accuracy 3]. A correct modeling of the quantum noise of a $\mathrm{GW}$ interferometer should take into account correlations between the two types of noises, which may allow sub-SQL sensitivities to be achieved [3, 4, 5].

The need for optical field simulation for gravitationalwave interferometer design has been addressed in the past with a variety of simulation tools, both in the frequency domain (e.g., twiddle [6] and finesse [7]) and in the time domain (e.g., the LIGO end-to-end simulation program [8]). Although time-domain simulations can study issues associated with large mirror displacements and non-linear effects, e.g., the lock acquisition of the interferometer, they are computationally costly; in addition, full time-domain simulations are also less straightforward to quantize. In order to study the performance of gravitational-wave detectors, it suffices to stay in the linear regime near the operation point. For such a linear problem, frequency-domain simulations are dramatically simpler than time-domain ones; it is straightforward to obtain frequency-domain transfer functions, and therefore noise spectra. In addition, since the system is linear, the propagation of quantum Heisenberg operators are identical to those of classical field amplitudes, therefore it suffices to build an essentially classical propagator.

In low-power situations where radiation-pressureinduced mirror motion is negligible and no non-linear op- tical elements (e.g., squeezers) are used, when linearizing over mirror displacements, propagation of electromagnetic fields at different frequencies are independent, and therefore the transfer functions can be established for each different frequency separately. One only needs to take into account that, for the inputs to this linear system: (i) mirror motion (with frequency $\Omega$ ) creates phase modulation of the carrier, which is equivalent to generating two equally spaced sidebands on the carrier frequency (at $\omega \pm \Omega$, where $\omega$ is the carrier frequency and we denote $\omega+\Omega$ and $\omega-\Omega$ as the upper and lower sidebands, respectively) with opposite amplitudes, and that (ii) laser noise can usually be decomposed into amplitude noise and phase noise, with the former contributing equally to the upper and lower sidebands, and the latter oppositely. These considerations have been the conceptual foundations of previous frequency-domain simulation programs.

For high-power interferometers, the above strategy will have to be modified: the radiation-pressure forces acting on the mirrors, at frequency $\Omega$, depend on both upper and lower sideband fields; the induced mirror motion will again contribute to both sidebands - this makes it necessary to propagate pairs of upper and lower sidebands simultaneously. The mathematical formalism most convenient for this problem, at least in the case of only one carrier frequency, is the Caves-Schumaker two-photon formalism [9, 10. In this paper, we adopt this formalism and present a mathematical framework for calculating the propagation of fields in an arbitrary optical system that includes the dynamical response of the mirrors to the light field. Namely, we divide complex interferometers into inter-connected elementary subsystems, and provide a general procedure for building a set of linear equations for all optical fields propagating between these systems - based on each individual system's input-output relation, i.e., transformation matrices relating output fields to input ones and the incoming GW. We also describe the way in which these subsystems are connected to each other. Solving these equations will provide us with the optical fields, in terms of vacuum fluctuations entering the system from open ports, laser noise, and incoming GWs. While this mathematical framework, and the resulting numerical simulation tool, were developed to model quantum correlation effects in gravitational-wave 

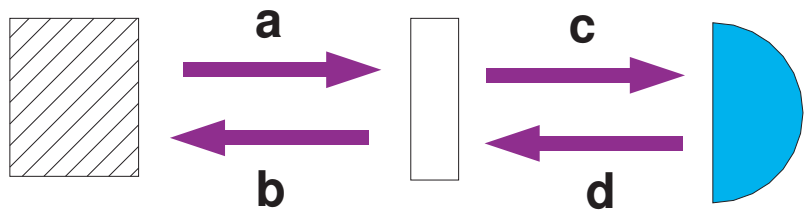

FIG. 1: A sample configuration is shown. A beam block is connected to a mirror, which is in turn connected to a detector. Input fields incident on the mirror, $\mathbf{a}$ and $\mathbf{d}$, are related to the output fields, $\mathbf{b}$ and $\mathbf{c}$, by matrix operators derived in Sections III and IV

interferometers, the method is general and can be used in any system where optical fields couple to mechanical oscillation modes.

The paper is organized as follows: In Sec. I we introduce the mathematical framework for the simulation, and illustrate it with a simple example; in Sec. III we provide input-output relations of basic optical elements that may be present in a laser interferometer, ignoring radiationpressure effects and the presence of gravitational waves by re-formatting well-known results in optics; in Sec. IV] we take radiation-pressure-induced mirror motion into account, and provide input-output relations for movable mirrors and beamsplitters (up to linear order in mirror motion), which have not been obtained before in the most general form; in Sec. $\mathbf{\nabla}$ we take into account the presence of GWs by introducing modulation of cavity lengths, and treat the corresponding effect on light propagation up to linear order in $L / \lambda_{\mathrm{GW}}$ (with $L$ the length of the interferometer). In Sec. $\nabla \mathbb{1}$ the formulation is applied to a novel interferometer designed to extract squeezed vacuum states that are created by a strong opto-mechanical coupling; and, finally, conclusions are summarized in Section VII

\section{MATHEMATICAL FRAMEWORK}

\section{A. General Prescription}

As mentioned above, the presence of opto-mechanical coupling dictates that we propagate the upper and lower sidebands simultaneously, which means that for each frequency $\Omega$, we will have to work with the two-dimensional linear space spanned by the upper $[a(\omega+\Omega)]$ and lower $[a(\omega-\Omega)]$ sidebands [16]. Within the two-photon formalism, developed by Schumaker and Caves [9, 10], and outlined in Appendix $\AA$ below, instead of $a(\omega \pm \Omega)$, the two quadrature fields $a_{1,2}(\Omega)$ are chosen as the basis vectors. For simplicity of notation, we generally denote

$$
\mathbf{a} \equiv\left(\begin{array}{l}
a_{1} \\
a_{2}
\end{array}\right)
$$

and suppress the dependence of $\mathbf{a}$ on $\Omega$.
We consider optomechanical systems formed by the following elementary subsystems: movable mirrors, beamsplitters and free space propagators. We will also include a "linear squeezer", which turns an ordinary vacuum state into a two-mode squeezed field with arbitrary squeeze factor and squeeze angle. Auxiliary to these optical elements, we introduce the beam block and the photodetector to deal with open ports which are either left undetected and those detected with unit quantum efficiency; we also introduce the laser as an optical element, which injects monochromatic carrier light and laser noise into the interferometer. Quadrature optical fields undergo linear transformations when propagating through such elementary systems, and quadrature fields with different $\Omega$ 's propagate independently from each other. These linear transformations are described mathematically by the input-output relation, namely a set of equations relating the output fields to the input ones, including vacuum fluctuations, the carrier laser and laser fields, as well as to incoming GWs. We provide these input-output relations in Secs. IIID

However, we note that propagation of sideband quadratures $(\Omega \neq 0)$, although independent from each other, all depend on the propagation of the carrier quadratures $(\Omega=0)$, i.e., the amplitude and phase of the carrier incident on each subsystem. Fortunately, the propagation of the carrier is not affected by that of the sidebands, and can be carried out independently at the beginning. This said, we begin to formulate our general method of simulation.

We build the following system of linear equations (for each sideband frequency $\Omega$ )

$$
\left[\begin{array}{ccc}
\mathcal{M}_{11} & \cdots & \mathcal{M}_{1 N} \\
\vdots & \cdots & \vdots \\
\mathcal{M}_{N 1} & \cdots & \mathcal{M}_{N N}
\end{array}\right]\left[\begin{array}{c}
\mathbf{a}^{(1)} \\
\vdots \\
\mathbf{a}^{(N)}
\end{array}\right]=\left[\begin{array}{c}
\mathbf{u}^{(1)} \\
\vdots \\
\mathbf{u}^{(N)}
\end{array}\right]
$$

where $\mathbf{a}^{(i)}, i=1, \ldots, N$ are the $N$ quadrature fields (each of them a two-dimensional vector) propagating in every part of the system, $\mathbf{u}^{(i)}, i=1, \ldots N$ are $N$ generalized input quadrature fields (each of them again a two-dimensional vector). The $\mathcal{M}_{i j}, i, j=1, \ldots N$ are $2 \times 2$ matrices which depend on the details of the optical system, and the $\mathbf{u}^{(i)}$ can be written schematically as

$$
\mathbf{u}^{(i)}=\mathbf{v}^{(i)}+\mathbf{l}^{(i)}+\mathbf{H}^{(i)} h,
$$

where $\mathbf{v}^{(i)}$ arises from vacuum fluctuations entering from the detection port or other lossy ports (Secs. [B] III) and IV), $\mathbf{l}^{(i)}$ from the laser (Sec. IIB), and $\mathbf{H}^{(i)} h$ from GWinduced phase modulation, with $h$ the GW amplitude (Sec. V); depending on the location of this generalized input field, some or all of the above three contributions could also be zero. Henceforth in the paper, we shall consider each pair of quadrature fields as one object. Inverting the matrix $\mathcal{M}_{i j}$ will give $\mathbf{a}^{(i)}$ in terms of $\mathbf{u}^{(i)}$, and hence all of the necessary transfer functions.

Now let us provide a universal prescription for constructing Eq. (2), suitable for modelling generic systems. 
We break this procedure into two steps:

1. Suppose we have $n$ elementary subsystems mentioned above, with the $k^{\text {th }}$ subsystem having $p_{k}$ ports. The entire system will then have $P \equiv$ $\sum_{k=1}^{n} p_{k}$ ports. Because we formally include beam blocks and photodetectors as subsystems, none of our ports will be formally open, i.e., left unconnected to some other port. This means that we have $P / 2$ pairs of connections. For each pair of connections, we have two fields, one propagating in each direction. This means we have a total of $P$ fields (each in turn has two quadrature components).

2. For each system $k$, with $p_{k}$ ports, we also have $p_{k}$ input fields and $p_{k}$ output fields, and therefore the input-output relation will provide us $p_{k}$ equations. All subsystems together will then provide us with $P$ equations (each with two components), exactly the number needed.

\section{B. Example with the input-output relation of beam blocks, photodetectors and lasers}

Next we illustrate the generic construction procedure with a simple example, which also clarifies the formal roles of beam blocks, photodetectors, and lasers. We first propagate fields between three basic elements of an optical train: a beam block, a partially reflecting mirror, and a photodetector. Referring to Fig. [ the beam block is connected to the mirror, which is in turn connected to a detector. For simplicity, we assume that the mirror is lossless and fixed in position.

As a first step, we identify the fields in consideration. The beam block and the photodetector are 1-port systems, the mirror is a 2-port system; we have a total of 4 ports, and $4 / 2=2$ connections. There are two fields associated with each connection; we label them $\mathbf{a}, \mathbf{b}$, and c, d, respectively, as done in Fig. 1 Note that each field in turn has two quadrature components, so the system is 8 -dimensional, and we need 8 scalar equations.

Now we have to provide the input-output relations for each object. For the mirror with amplitude reflectivity $\rho$ and transmissivity $\tau$, and neglecting radiation pressure effects, we have

$$
\left(\begin{array}{l}
\mathbf{b} \\
\mathbf{c}
\end{array}\right)=\left(\begin{array}{cc}
-\rho & \tau \\
\tau & \rho
\end{array}\right)\left(\begin{array}{l}
\mathbf{a} \\
\mathbf{d}
\end{array}\right) \equiv \mathbf{M}_{\mathrm{Mir}}\left(\begin{array}{l}
\mathbf{a} \\
\mathbf{d}
\end{array}\right) .
$$

Note that Eq. (4) contains 4 scalar equations, and that $\rho$ and $\tau$ are really $2 \times 2$ scalar matricies, $\rho \mathbf{I}$, and $\tau \mathbf{I}$ (this is true because our mirror does not mix quadratures) we have suppressed the identity matrix I for simplicity.
To comply with the format of Eq. (2), we write

$$
\left(\begin{array}{cccc}
-\rho & -1 & 0 & \tau \\
\tau & 0 & -1 & \rho
\end{array}\right)\left(\begin{array}{l}
\mathbf{a} \\
\mathbf{b} \\
\mathbf{c} \\
\mathbf{d}
\end{array}\right)=\left(\begin{array}{l}
0 \\
0
\end{array}\right)
$$

For the beam block and the photodetector, they really are placeholders for physically open ports. Their inputoutput relation is simply that the output fields from them are vacuum fluctuations (independent from the input fields):

$$
\mathbf{a}=\mathbf{v}^{(1)}, \quad \mathbf{d}=\mathbf{v}^{(2)},
$$

Here we assume implicitly that the photodetector is detecting the field $\mathbf{c}$ with unit quantum efficiency. In order to model imperfect photodetectors, we could add a mirror with zero reflectivity and non-zero loss in front of the ideal photodetector.

Combining Eqs. (5) and (6), we have

$$
\underbrace{\left(\begin{array}{cccc}
-1 & 0 & 0 & 0 \\
-\rho & -1 & 0 & \tau \\
\tau & 0 & -1 & \rho \\
0 & 0 & 0 & -1
\end{array}\right)}_{\mathcal{M}}\left(\begin{array}{l}
\mathbf{a} \\
\mathbf{b} \\
\mathbf{c} \\
\mathbf{d}
\end{array}\right)=\left(\begin{array}{c}
-\mathbf{v}^{(1)} \\
0 \\
0 \\
-\mathbf{v}^{(2)}
\end{array}\right),
$$

which are the 8 scalar equations we need. Inverting $\mathcal{M}$ will give us each of the propagating fields in terms of the input vacuum fields.

Now suppose the beam block is replaced by a laser source, coupled to the spatial mode of a field, then we only need to replace the vacuum field $\mathbf{v}^{(1)}$ in Eqs. (6) and (17) by the laser field, $\mathbf{l}^{(1)}$ : at $\Omega=0$, carrier quadratures, while at $\Omega \neq 0$, it gives the laser noises.

Here we note that all diagonal elements of $\mathcal{M}$ are equal to -1 - this is in fact not a coincidence, but a universal feature of our construction procedure. In order to understand this, we need to realize that every field $\mathbf{a}^{(k)}$ is the output field of exactly one subsystem. In the inputoutput relation of that unique subsystem, there is exactly one line that relates $\mathbf{a}^{(k)}$ to the input fields of this subsystem, which reads:

$$
\mathbf{a}^{(k)}=\left[\text { terms not involving } \mathbf{a}^{(k)}\right] .
$$

This equation corresponds to, after moving $\mathbf{a}^{(k)}$ to the right-hand side of the equation, moving any non-a $\mathbf{a}^{(j)}, j=$ $1, \ldots, N$ terms to the left-hand side, and swapping left and right,

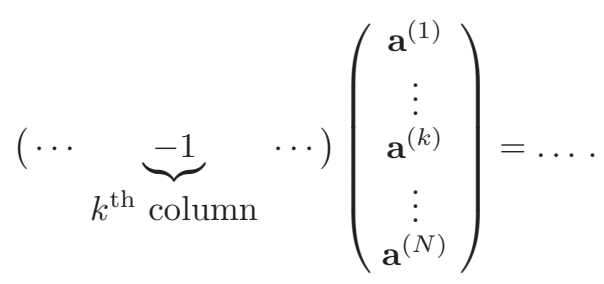


It is obvious that the lines of equation found by this way for different $\mathbf{a}^{(k)}$ 's will be different. As a consequence, we can arrange to have the line corresponding to $\mathbf{a}^{(k)}$ appear on the $k^{\text {th }}$ row of $\mathcal{M}$, and thus have all its diagonal elements equal to -1 .

\section{MATRICES FOR STATIC OPTICAL ELEMENTS}

In this section, we derive the matrices for some standard objects used in simulating quantum noise in a gravitational-wave interferometer. Here we neglect radiation pressure effects and the presence of gravitational waves (they will be dealt with in Secs. IV and $\nabla$ respectively). As a consequence, our derivation only involves some re-formatting of previously well-known results.

\section{A. Mirrors}

Field transformations due to a mirror were introduced in the example of Section III The transformation matrix for a lossless mirror is given in Eq. (4). We now derive more complete equations for the mirror that include losses. We ascribe a power loss $A$ to the mirror in Fig. 11 such that $\rho^{2}+\tau^{2}+A=1$. The introduction of losses gives rise to an additional vacuum field of amplitude $\sqrt{A /(1-A)}$ that is added to each input of the mirror. The $(1-A)^{-1}$ factor accounts for part of the loss field being lost to the mirror. This can be verified by having shot-noise-limited fields, a and $\mathbf{d}$, incident on the mirror. The field returning to the beam block

$$
-\rho\left(\mathbf{a}+\sqrt{\frac{A}{1-A}} \mathbf{v}^{(3)}\right)+\tau\left(\mathbf{d}+\sqrt{\frac{A}{1-A}} \mathbf{v}^{(4)}\right)
$$

must also be at the shot noise level, such that

$$
\rho^{2}\left(1+\frac{A}{1-A}\right)+\tau^{2}\left(1+\frac{A}{1-A}\right)=\frac{1-A}{1-A}=1
$$

The new equations governing the mirror are

$$
\left(\begin{array}{l}
\mathbf{b} \\
\mathbf{c}
\end{array}\right)=\left(\begin{array}{cc}
-\rho & \tau \\
\tau & \rho
\end{array}\right)\left(\begin{array}{c}
\mathbf{a}+\sqrt{\frac{A}{1-A}} \mathbf{v}^{(3)} \\
\mathbf{d}+\sqrt{\frac{A}{1-A}} \mathbf{v}^{(4)}
\end{array}\right)
$$

where $\mathbf{v}^{(3)}$ and $\mathbf{v}^{(4)}$ are the vacuum fluctuations that enter due to the presence of loss.

Equation (12) may be rewritten as

$$
\left(\begin{array}{l}
\mathbf{b} \\
\mathbf{c}
\end{array}\right)=\left(\begin{array}{cc}
-\rho & \tau \\
\tau & \rho
\end{array}\right)\left(\begin{array}{l}
\mathbf{a} \\
\mathbf{d}
\end{array}\right)+\sqrt{A}\left(\begin{array}{c}
\mathbf{v}^{(3) \prime} \\
\mathbf{v}^{(4) \prime}
\end{array}\right),
$$

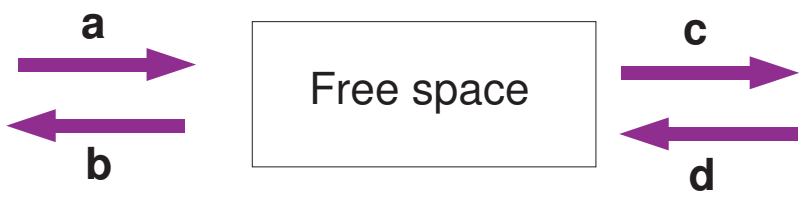

FIG. 2: The fields entering and exiting a region of free space are shown. Propagation operators are characterized by the propagation distance (and orientation relative to the source polarization, in the case of the GW signal).

where

$$
\begin{aligned}
\mathbf{v}^{(3) \prime} & \equiv \sqrt{\frac{1}{1-A}}\left(-\rho \mathbf{v}^{(3)}+\tau \mathbf{v}^{(4)}\right) \\
\mathbf{v}^{(4) \prime} & \equiv \sqrt{\frac{1}{1-A}}\left(\tau \mathbf{v}^{(3)}+\rho \mathbf{v}^{(4)}\right)
\end{aligned}
$$

$\mathbf{v}^{(3) \prime}$ and $\mathbf{v}^{(4) \prime}$ are uncorrelated vacuum fields in this representation. We can subsequently write the mirror's contribution to Eq. (2) as

$$
\left(\begin{array}{cccc}
-\rho & -1 & 0 & \tau \\
\tau & 0 & -1 & \rho
\end{array}\right)\left(\begin{array}{l}
\mathbf{a} \\
\mathbf{b} \\
\mathbf{c} \\
\mathbf{d}
\end{array}\right)=\left(\begin{array}{c}
-\sqrt{A} \mathbf{v}^{(3) \prime} \\
-\sqrt{A} \mathbf{v}^{(4) \prime}
\end{array}\right)
$$

This method may also be used to inject losses in beamsplitters or cavities.

\section{B. Free space propagation}

Since optical cavities are present in virtually all optical configurations of gravitational-wave interferometers, we must give a transformation matrix for them as an element of our arbitrary optical train. To do so we introduce an operator to transform the field as it propagates through free space between any two other optical elements (in the case of an optical cavity, these would be mirrors). Using the convention of Fig. 2 the matrix for propagation through a length $L$ transforms input fields a and $\mathbf{d}$ according to

$$
\left(\begin{array}{l}
\mathbf{b} \\
\mathbf{c}
\end{array}\right)=\mathbf{M}_{\text {Prop }}\left(\begin{array}{l}
\mathbf{a} \\
\mathbf{d}
\end{array}\right)
$$

where the matrix for the propagator is

$$
\mathbf{M}_{\text {Prop }} \equiv e^{i \phi}\left(\begin{array}{cc}
0 & \mathbf{R}_{\Theta} \\
\mathbf{R}_{\Theta} & 0
\end{array}\right) \text {. }
$$

Here

$$
\begin{aligned}
\Theta & \equiv \frac{\omega L}{c}, \\
\phi & \equiv \frac{\Omega L}{c},
\end{aligned}
$$




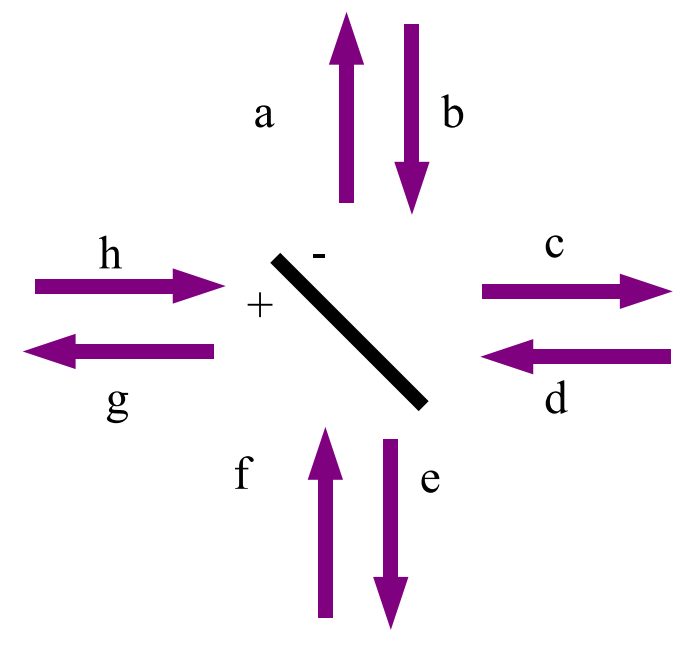

FIG. 3: Treating the beamsplitter as a four-port device, definitions for the fields, including sign conventions, are shown.

are the one-way phase shift on the carrier light at frequency, $\omega$, and on modulation sidebands at frequency, $\Omega$, respectively, and

$$
\mathbf{R}_{\Theta} \equiv\left(\begin{array}{cc}
\cos \Theta & -\sin \Theta \\
\sin \Theta & \cos \Theta
\end{array}\right)
$$

is the rotation operator on quadrature fields.

\section{Beamsplitters}

Another essential optical element of an interferometer is the beamsplitter. We consider a beamsplitter with amplitude reflectivity and transmissivity, $\rho$ and $\tau$, respectively. The beamsplitter transforms the input fields, shown in Figure 3 according to the matrix equation

$$
\left(\begin{array}{l}
\mathbf{a} \\
\mathbf{c} \\
\mathbf{e} \\
\mathbf{g}
\end{array}\right)=\mathbf{M}_{B S}\left(\begin{array}{l}
\mathbf{d} \\
\mathbf{b} \\
\mathbf{h} \\
\mathbf{f}
\end{array}\right)
$$

where

$$
\mathbf{M}_{B S} \equiv\left(\begin{array}{cccc}
-\rho & 0 & 0 & \tau \\
0 & -\rho & \tau & 0 \\
0 & \tau & \rho & 0 \\
\tau & 0 & 0 & \rho
\end{array}\right)
$$

In presence of optical loss, assuming $\rho^{2}+\tau^{2}+A=1$, and going through similar arguments to Sec. III we simply add a column vector of vacuum fields $-\sqrt{A} \mathbf{v}^{(i)}$ $(i=1,2,3,4)$ onto the right-hand side of Eq. (22).

\section{Correlators}

The correlator module of the simulation allows for the inclusion of squeezed light or vacuum fields in the interferometer. It is essentially a one-way device: only fields entering from one direction are transformed; fields entering from the other direction pass through the correlator unmodified. Taking a to be the input field, the field at the output of the correlator, $\mathbf{b}$, is defined by

$$
\mathbf{b}=\mathbf{S}(r, \phi) \mathbf{a},
$$

where $\mathbf{S}(r, \phi)$ is the squeeze operator with squeeze factor $r$ and squeeze angle $\phi$ :

$$
\begin{aligned}
& \mathbf{S}(r, \phi) \equiv \\
& \left(\begin{array}{cc}
\cosh r+\sinh r \cos 2 \phi & \sinh r \sin 2 \phi \\
\sinh r \sin 2 \phi & \cosh r-\sinh r \cos 2 \phi
\end{array}\right) .
\end{aligned}
$$

\section{RADIATION PRESSURE}

Radiation pressure plays an important role in interferometers operating close to or beyond the SQL, since quantum back-action noise must be taken into account. Moreover, radiation-pressure effects can also modify the dynamics of these interferometers [5]. Sideband quadrature fields create amplitude modulations to the carrier field, and the associated power modulation drives the motion of optical elements, which, in turn, phase modulates the carrier, thereby creating sideband quadrature fields.

Details of this sideband-to-sideband conversion depend on the phases (this determines which quadrature gets converted into which) and amplitudes (this determines the conversion strength) of the carrier field propagating in different parts of the interferometer. Therefore, it is necessary to separate the fields into carrier $(\Omega=0)$ and sideband $(\Omega \neq 0)$ components at this point. The radiation pressure force due to the carrier field itself is a time independent force and can be ignored (in reality they will be balanced by a static force exerted on the optical elements, e.g., the pendulum restoring force on a suspended mirror). The effect of interest is the time-dependent part of the force, due to sideband components, which will be the subject of this section. As a foundation, we must first of all calculate the phase and amplitude of the carrier fields at each location. But this we can already do by building the general equation (2) out of input-output relations of static optical elements, which have already been derived in Sec. III and solving it.

Before incorporating radiation pressure into the treatment of specific systems, let us study the electromagnetic momentum flux carried by optical fields in the twophoton formalism. In quadrature representation, we decompose the total quadrature field $\mathbf{E}_{j}^{\text {total }}$ (here $\mathbf{E}_{j}$ can be $\mathbf{a}, \mathbf{b}, \mathbf{c}$ or $\mathbf{d}$ for the configuration in Fig. (1) into the 
following two terms:

$$
\mathbf{E}_{j}^{\text {total }}=\mathbf{E}_{j}^{\text {carrier }}+\mathbf{E}_{j}^{\mathrm{sb}} .
$$

The monochromatic carrier field in Eq. (26) can be written more explicitly in terms of power $I_{j}$, phase $\theta_{j}$ and effective beam area $\mathcal{A}$ as

$$
\mathbf{E}_{j}^{\text {carrier }}=\sqrt{\frac{8 \pi I_{j}}{\mathcal{A c}}}\left(\begin{array}{c}
\cos \theta_{j} \\
\sin \theta_{j}
\end{array}\right),
$$

while the sideband field can be written as an integral over all sideband frequencies:

$$
\mathbf{E}_{j}^{\mathrm{sb}}(t)=\sqrt{\frac{4 \pi \hbar \omega}{\mathcal{A} c}} \int_{0}^{+\infty} \frac{d \Omega}{2 \pi}\left[\mathbf{j}(\Omega) e^{-i \Omega t}+\text { H.c. }\right] .
$$

The total momentum flow carried by the field is

$$
\frac{\mathcal{A}}{4 \pi}\left(\mathbf{E}_{j}^{\text {carrier }}+\mathbf{E}_{j}^{\mathrm{sb}}\right)^{2} .
$$

Removing the static $(\mathrm{dc})$ and optical frequency $(\omega)$ components, the Fourier transform of the time-averaged (over a time scale much shorter than the GW period, but much longer than $1 / \omega$ ) ac momentum flow carried by this field is

$$
\dot{P}_{j}(\Omega)=\sqrt{\frac{\hbar \omega}{c^{2}}} \mathbf{D}_{j}^{T} \mathbf{j}(\Omega),
$$

where we have defined

$$
\mathbf{D}_{j} \equiv \sqrt{\frac{\mathcal{A} c}{4 \pi}} \mathbf{E}_{j}^{\text {carrier }}=\sqrt{2 I_{j}}\left(\begin{array}{c}
\cos \theta_{j} \\
\sin \theta_{j}
\end{array}\right)
$$

as the carrier quadrature field, and $\mathbf{j}(\Omega)$ is the sideband component at angular frequency $\Omega$.

In the remainder of this Section we derive explicit input-output relations for mirrors and beamsplitters, including radiation pressure effects. Our results will be more general than previously obtained results by allowing the carrier fields incident from different ports to have different phases.

\section{A. Mirrors}

Let us once again consider the mirror in Fig. 11 Assuming that the mirror behaves as a free particle with mass $M$ when no radiation-pressure forces are exerted (valid for suspended mirrors when frequencies greater than the pendulum resonant frequency are considered), the Fourier transform for the equation of motion for the mirror is

$$
-M \Omega^{2} X=\sum_{j} \eta_{j} \dot{P}_{j}
$$

where $\mathrm{X}$ is the displacement of the mirror induced by all the sideband fields ( $X$ is positive to the left in Fig. 1 and the $j$ refer to $\mathbf{a}, \mathbf{b}, \mathbf{c}, \mathbf{d})$. The summation is performed over all the fields entering and exiting the mirror; the coefficients $\eta_{a}=\eta_{b}=-1$ and $\eta_{c}=\eta_{d}=1$ account for the directions of propagation. The displacement of the mirror due to the radiation pressure forces, $X$, can be written explicitly as [see Eq. [30]

$$
\begin{aligned}
X=\frac{1}{M \Omega^{2}} \sqrt{\frac{\hbar \omega}{c^{2}}} & {\left[\left(\begin{array}{ll}
\mathbf{D}_{a}^{T} & -\mathbf{D}_{d}^{T}
\end{array}\right)\left(\begin{array}{l}
\mathbf{a} \\
\mathbf{d}
\end{array}\right)\right.} \\
& \left.+\left(\begin{array}{ll}
\mathbf{D}_{b}^{T} & -\mathbf{D}_{c}^{T}
\end{array}\right)\left(\begin{array}{l}
\mathbf{b} \\
\mathbf{c}
\end{array}\right)\right] .
\end{aligned}
$$

Given a (time-dependent) displacement $X(t)$ of the mirror, the input-output relation can be written as (if $\dot{X} \ll$ c)

$$
\begin{aligned}
& E_{b}^{\text {total }}(t)=-\rho E_{a}^{\text {total }}\left[t+\frac{2 X(t)}{c}\right]+\tau E_{d}^{\text {total }}(t) \\
& E_{c}^{\text {total }}(t)=\tau E_{a}^{\text {total }}(t)+\rho E_{d}^{\text {total }}\left[t-\frac{2 X(t)}{c}\right] .
\end{aligned}
$$

$c$ in the argument of $E_{j}^{\text {total }}$ for the $j$-th field is the speed of light and should be distinguished from $c$ in the subscript of $E_{j}^{\text {total }}$, which refers to the field c. In quadrature representation, to leading order in $X$ and in the sideband field amplitudes, we have,

$$
\begin{aligned}
& E_{j}^{\text {total }}\left[t \mp \frac{2 X(t)}{c}\right] \\
\Leftrightarrow & \mathbf{E}_{j}^{\text {carrier }}+\mathbf{E}_{j}^{\mathrm{sb}}(t) \pm \frac{2 \omega X(t)}{c} \mathbf{R}_{\Theta=\pi / 2} \mathbf{E}_{j}^{\text {carrier }}, \\
= & \mathbf{E}_{j}^{\text {carrier }}+\mathbf{E}_{j}^{\mathrm{sb}}(t) \mp \frac{2 \omega X(t)}{c}\left[\mathbf{E}_{j}^{\text {carrier }}\right]^{*} .
\end{aligned}
$$

Here ${ }^{*}$ refers to a rotation by $\pi / 2$, as described by $-\mathbf{R}_{\Theta=\pi / 2}$ in Eq. (35). Accordingly, for any quadrature field $\mathbf{v}$, we define

$$
\mathbf{v}^{*} \equiv\left(\begin{array}{c}
v_{2} \\
-v_{1}
\end{array}\right), \quad \text { for } \mathbf{v}=\left(\begin{array}{c}
v_{1} \\
v_{2}
\end{array}\right) .
$$

Equation (35) implies that time delays, or phase modulations, create sideband quadratures orthogonal to the

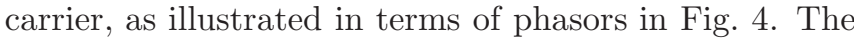
sideband part, i.e., the ac components in Eqs. (34a) and (34b), can be obtained using Eq. (35):

$$
\left(\begin{array}{l}
\mathbf{b} \\
\mathbf{c}
\end{array}\right)=\mathbf{M}_{\text {mirror }}\left(\begin{array}{l}
\mathbf{a} \\
\mathbf{d}
\end{array}\right)-\frac{2 \rho \omega X}{c \sqrt{\hbar \omega}}\left(\begin{array}{l}
\mathbf{D}_{a}^{*} \\
\mathbf{D}_{d}^{*}
\end{array}\right) .
$$

Inserting Eq. (33) into Eq. (37) gives

$$
\begin{aligned}
& {\left[\mathbf{I}+\Pi\left(\begin{array}{l}
\mathbf{D}_{a}^{*} \\
\mathbf{D}_{d}^{*}
\end{array}\right)\left(\begin{array}{ll}
\mathbf{D}_{b}^{T} & -\mathbf{D}_{c}^{T}
\end{array}\right)\right]\left[\begin{array}{l}
\mathbf{b} \\
\mathbf{c}
\end{array}\right] } \\
= & {\left[\mathbf{M}_{\text {mirror }}-\Pi\left(\begin{array}{l}
\mathbf{D}_{a}^{*} \\
\mathbf{D}_{d}^{*}
\end{array}\right)\left(\begin{array}{ll}
\mathbf{D}_{a}^{T} & -\mathbf{D}_{d}^{T}
\end{array}\right)\right]\left[\begin{array}{l}
\mathbf{a} \\
\mathbf{d}
\end{array}\right] . }
\end{aligned}
$$




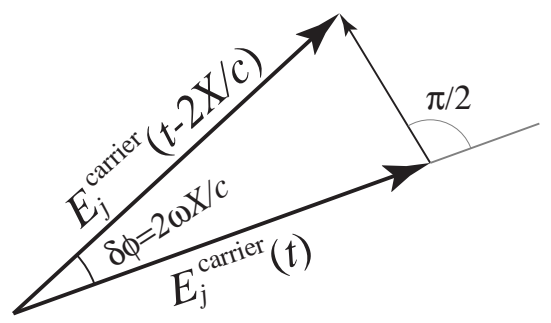

FIG. 4: Here we show that the phase modulation sideband generated by the radiation pressure force is perpendicular to the carrier field, which is why the generated signal has a $\mathbf{D}^{*}$ dependence.

where

$$
\Pi \equiv \frac{2 \rho \omega}{M \Omega^{2} c^{2}}
$$

is a quantity with units of inverse power or $\mathrm{W}^{-1}$. [For lossy mirrors with $\rho^{2}+\tau^{2}+A=1$, we simply insert a column vector $-\sqrt{A} \mathbf{v}^{(i)}, i=1,2$ onto the right-hand sides of Eq. [37] and (38), Cf. Sec. [IIA]

To solve for $\mathbf{b}$ and $\mathbf{c}$, the matrix on the left hand side of Eq. (38) must be inverted. It is straightforward to find a complete set of eigenvectors for this matrix, they are:

$$
\left[\boldsymbol{\xi}_{1}, \boldsymbol{\xi}_{2}, \boldsymbol{\xi}_{3}, \boldsymbol{\xi}_{4}\right]=\left[\left(\begin{array}{c}
\mathbf{D}_{b}^{*} \\
0
\end{array}\right),\left(\begin{array}{c}
0 \\
\mathbf{D}_{c}^{*}
\end{array}\right),\left(\begin{array}{c}
\mathbf{D}_{c} \\
\mathbf{D}_{b}
\end{array}\right),\left(\begin{array}{c}
\mathbf{D}_{a}^{*} \\
\mathbf{D}_{d}^{*}
\end{array}\right)\right] .
$$

Since the first three vectors are orthogonal to $\left(\begin{array}{ll}\mathbf{D}_{b}^{T} & -\mathbf{D}_{c}^{T}\end{array}\right)$, the three corresponding eigenvalues are $\lambda_{1}=\lambda_{2}=\lambda_{3}=1$; the last eigenvalue is

$$
\begin{aligned}
\lambda_{4} & =1+\Pi\left[\mathbf{D}_{b}^{T} \mathbf{D}_{a}^{*}-\mathbf{D}_{c}^{T} \mathbf{D}_{d}^{*}\right] \\
& =1+2 \tau \Pi \mathbf{D}_{d}^{T} \mathbf{D}_{a}^{*} \\
& =1+\frac{8 \rho \tau \omega \sqrt{I_{a} I_{d}}}{M \Omega^{2} c^{2}} \sin \left(\theta_{a}-\theta_{d}\right) .
\end{aligned}
$$

Inverting the eigenvalue $\lambda_{4}$ yields a pair of resonant frequencies at

$$
\pm \Omega_{\mathrm{M}}= \pm\left[\frac{-8 \rho \tau \omega \sqrt{I_{a} I_{d}}}{M c^{2}} \sin \left(\theta_{a}-\theta_{d}\right)\right]^{1 / 2}
$$

Physically, this resonance comes about because the sideband fields generated by mirror motion can exert radiation pressure back onto the mirror. Let us for a moment consider classical motion of the mirror. As was mentioned after Eq. (37), for any given input carrier field, the sideband field generated upon reflection from the moving mirror is $\pi / 2$ phase shifted relative to the input carrier, so the sideband will not beat with the reflected carrier to induce any force on the mirror [see Eq. [30] — force can only be induced by beating this motion-induced sideband field with the transmitted carrier, which must have nonzero amplitude and must have a phase difference other than $\pi / 2$ relative to the sideband. This explains why the resonant frequency vanishes if either $\rho=0$ or $\tau=0$ (no reflected or transmitted field), or if $\theta_{a}-\theta_{d}=N \pi$ (no phase difference between the two input fields).

When the two input carrier fields, $\mathbf{D}_{a}$ and $\mathbf{D}_{d}$, have the same phase (or differ by $N \pi$ ), the phasors corresponding to $\mathbf{D}_{a}, \mathbf{D}_{b}, \mathbf{D}_{c}$ and $\mathbf{D}_{d}$ all become parallel to each other. This is true for almost all interferometers that have been treated explicitly analytically. This case is rather special from a mathematical point of view, since the matrix we are inverting does not have a complete set of eigenvectors. Fortunately, the inverse is just

$$
\begin{aligned}
& {\left[\mathbf{I}+\Pi\left(\begin{array}{l}
\mathbf{D}_{a}^{*} \\
\mathbf{D}_{d}^{*}
\end{array}\right)\left(\begin{array}{ll}
\mathbf{D}_{b}^{T} & -\mathbf{D}_{c}^{T}
\end{array}\right)\right]^{-1} } \\
= & {\left[\mathbf{I}-\Pi\left(\begin{array}{l}
\mathbf{D}_{a}^{*} \\
\mathbf{D}_{d}^{*}
\end{array}\right)\left(\begin{array}{ll}
\mathbf{D}_{b}^{T} & -\mathbf{D}_{c}^{T}
\end{array}\right)\right], \quad \text { if } \mathbf{D}_{a} \| \mathbf{D}_{d} ; }
\end{aligned}
$$

since

$$
\left[\left(\begin{array}{l}
\mathbf{D}_{a}^{*} \\
\mathbf{D}_{d}^{*}
\end{array}\right)\left(\begin{array}{ll}
\mathbf{D}_{b}^{T} & -\mathbf{D}_{c}^{T}
\end{array}\right)\right]^{2}=0, \quad \text { if } \mathbf{D}_{a} \| \mathbf{D}_{d}
$$

(This identity originates from the fact that the sideband field is orthogonal to the carrier field about which it is generated.) Using this fact, we can further simplify the input-output relation to

$$
\left(\begin{array}{c}
\mathbf{b} \\
\mathbf{c}
\end{array}\right)=\left[\mathbf{M}_{\text {mirror }}-2 \rho \Pi\left(\begin{array}{l}
\mathbf{D}_{a}^{*} \\
\mathbf{D}_{d}^{*}
\end{array}\right)\left(\begin{array}{ll}
\mathbf{D}_{a}^{T} & -\mathbf{D}_{d}^{T}
\end{array}\right)\left(\begin{array}{cc}
\rho & -\tau \\
\tau & \rho
\end{array}\right)\right]\left(\begin{array}{c}
\mathbf{a} \\
\mathbf{d}
\end{array}\right), \quad \text { if } \mathbf{D}_{a} \| \mathbf{D}_{d} .
$$

[Here for simplicity we have assumed the mirror to be lossless.] In practice, although Eq. (38) does not give the output fields $\mathbf{b}$ and $\mathbf{c}$ explicitly in terms of the input fields $\mathbf{a}$ and $\mathbf{d}$, it can be incorporated to the matrix $\mathcal{M}$ (and into $\mathbf{u}^{(i)}$, in presence of optical losses) without any trouble [cf. Eq. (2)]: its inversion will take place automatically when $\mathcal{M}^{-1}$ is calculated. [However, doing so will make it impossible to have -1 all along the diagonal of $\mathcal{M}$.] Alternatively, the variable $X$ may be added to our system of variables, with Eq. (33) providing the additional equation necessary. The equations governing a mirror may then be replaced with Eq. (37) to include the dependence on $X$. In this way, the -1 diagonal components are preserved, without the need to invert additional matrices. 


\section{B. Beamsplitter}

Referring to the fields shown in Fig. 3. the displacement due to radiation pressure forces on a beamsplitter (normal to its reflective face) is

$$
X_{\mathrm{N}}=\frac{X_{x}+X_{y}}{\sqrt{2}}=\frac{1}{M \Omega^{2}} \sqrt{\frac{\hbar \omega}{2 c^{2}}}\left[\left(\begin{array}{llll}
\mathbf{D}_{a}^{T} & \mathbf{D}_{c}^{T} & -\mathbf{D}_{e}^{T} & -\mathbf{D}_{g}^{T}
\end{array}\right)\left(\begin{array}{c}
\mathbf{a} \\
\mathbf{c} \\
\mathbf{e} \\
\mathbf{g}
\end{array}\right)+\left(\begin{array}{llll}
\mathbf{D}_{d}^{T} & \mathbf{D}_{b}^{T} & -\mathbf{D}_{h}^{T} & -\mathbf{D}_{f}^{T}
\end{array}\right)\left(\begin{array}{c}
\mathbf{d} \\
\mathbf{b} \\
\mathbf{h} \\
\mathbf{f}
\end{array}\right)\right]
$$

where $X_{x}$ is the displacement along the x-axis and $X_{y}$ is the displacement along the y-axis. Similar to the case of a cavity mirror, this motion induces phase fluctuations on the impinging fields upon reflection, and introduces additional terms in the input-output relation. Following a procedure similar to the one with which we obtain Eq. (37), we get

$$
\left(\begin{array}{l}
\mathbf{a} \\
\mathbf{c} \\
\mathbf{e} \\
\mathbf{g}
\end{array}\right)=\mathbf{M}_{\mathrm{BS}}\left(\begin{array}{c}
\mathbf{d} \\
\mathbf{b} \\
\mathbf{h} \\
\mathbf{f}
\end{array}\right)-\frac{\sqrt{2} \rho \omega X_{\mathrm{N}}}{c \sqrt{\hbar \omega}}\left(\begin{array}{c}
\mathbf{D}_{d}^{*} \\
\mathbf{D}_{b}^{*} \\
\mathbf{D}_{h}^{*} \\
\mathbf{D}_{f}^{*}
\end{array}\right)
$$

Inserting Eq. (46) into Eq. (47) gives

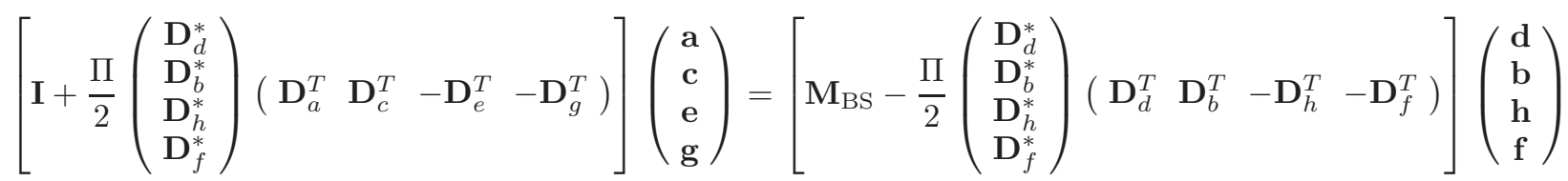

Equation (48) is quite similar in nature to Eq. (38); optical losses can also be incorporated in a similar fashion, by adding $-\sqrt{A} \mathbf{v}^{(i)}, i=1,2,3,4$ on to its right-hand side, where $\rho^{2}+\tau^{2}+A=1$. Again, in the generic case where

$$
\left(\begin{array}{llll}
\mathbf{D}_{a}^{T} & \mathbf{D}_{c}^{T} & -\mathbf{D}_{e}^{T} & -\mathbf{D}_{g}^{T}
\end{array}\right)\left(\begin{array}{c}
\mathbf{D}_{d}^{*} \\
\mathbf{D}_{b}^{*} \\
\mathbf{D}_{h}^{*} \\
\mathbf{D}_{f}^{*}
\end{array}\right) \neq 0
$$

the matrix on the LHS of Eq. (48) has eight linearly independent eigenvectors, of which seven have unit eigenvalue, while the eighth has

$$
\begin{aligned}
& \lambda_{8}=1+\frac{\Pi}{2}\left(\begin{array}{llll}
\mathbf{D}_{a}^{T} & \mathbf{D}_{c}^{T} & -\mathbf{D}_{e}^{T} & -\mathbf{D}_{g}^{T}
\end{array}\right)\left(\begin{array}{l}
\mathbf{D}_{d}^{*} \\
\mathbf{D}_{b}^{*} \\
\mathbf{D}_{h}^{*} \\
\mathbf{D}_{f}^{*}
\end{array}\right) \\
& =1+\tau \Pi\left(\mathbf{D}_{f}^{T} \mathbf{D}_{d}^{*}+\mathbf{D}_{h}^{T} \mathbf{D}_{b}^{*}\right) \\
& =1+\frac{4 \rho \tau \omega_{0}}{M \Omega^{2} c^{2}}\left[\sqrt{I_{f} I_{d}} \sin \left(\theta_{f}-\theta_{d}\right)+\sqrt{I_{h} I_{b}} \sin \left(\theta_{h}-\theta_{b}\right)\right] \text {, }
\end{aligned}
$$

which corresponds to an opto-mechanical resonance at angular frequency

$$
\pm \Omega_{\mathrm{BS}}= \pm\left\{-\frac{4 \rho \tau \omega}{M c^{2}}\left[\sqrt{I_{h} I_{b}} \sin \left(\theta_{h}-\theta_{b}\right)+\sqrt{I_{f} I_{d}} \sin \left(\theta_{f}-\theta_{d}\right)\right]\right\}^{1 / 2}
$$

In the special case of

$$
\left(\begin{array}{llll}
\mathbf{D}_{a}^{T} & \mathbf{D}_{c}^{T} & -\mathbf{D}_{e}^{T} & -\mathbf{D}_{g}^{T}
\end{array}\right)\left(\begin{array}{l}
\mathbf{D}_{d}^{*} \\
\mathbf{D}_{b}^{*} \\
\mathbf{D}_{h}^{*} \\
\mathbf{D}_{f}^{*}
\end{array}\right)=0
$$

i.e., all input carrier fields are in phase with each other (modulo $\pi$ ) we get

$$
\left(\begin{array}{l}
\mathbf{a} \\
\mathbf{c} \\
\mathbf{e} \\
\mathbf{g}
\end{array}\right)=\left[\mathbf{M}_{\mathrm{BS}}-\rho \Pi\left(\begin{array}{c}
\mathbf{D}_{d}^{*} \\
\mathbf{D}_{b}^{*} \\
\mathbf{D}_{h}^{*} \\
\mathbf{D}_{f}^{*}
\end{array}\right)\left(\begin{array}{llll}
\mathbf{D}_{d}^{T} & \mathbf{D}_{b}^{T} & -\mathbf{D}_{h}^{T} & -\mathbf{D}_{f}^{T}
\end{array}\right)\left(\begin{array}{cccc}
\rho & & & -\tau \\
& \rho & -\tau & \\
& \tau & \rho & \\
\tau & & & \rho
\end{array}\right)\right]\left(\begin{array}{c}
\mathbf{d} \\
\mathbf{b} \\
\mathbf{h} \\
\mathbf{f}
\end{array}\right), \quad \text { if } \mathbf{D}_{b}\left\|\mathbf{D}_{d}\right\| \mathbf{D}_{f} \| \mathbf{D}_{h}
$$


For simplicity, we assume the beamsplitter to be lossless in the above equation. This is particularly true for the beamsplitter in Michelson- and Sagnac-type GW interferometers 11]. Similar to the case of the mirror, for the purposes of simulation, we incorporate the position of the beamsplitter as an additional variable in $\mathcal{M}$, in order to preserve the -1 diagonal elements and to avoid the inversion of additional matrices.

\section{GRAVITATIONAL WAVE SIGNAL AND THE OUTPUT FIELD}

\section{A. GW contribution}

In our set of optical elements, only optical cavities have significant propagation distances, so we model the effect of GWs by introducing a phase shift to the carrier light as it passes through a cavity. To calculate the propagation of these fields, all that must be done is to add a source term in the equation governing the cavity. Refering to the fields in Fig. 2] the cavity field becomes

$$
\begin{aligned}
\mathbf{c} & =e^{i \phi} \mathbf{R}_{\Theta} \mathbf{a}-\eta \frac{\omega L h}{2 c \sqrt{\hbar \omega}} \mathbf{D}_{c}^{*} \\
& =\mathbf{R}_{\Theta}\left[e^{i \phi} \mathbf{a}-\eta \frac{\omega L h}{2 c \sqrt{\hbar \omega}} \mathbf{D}_{a}^{*}\right]
\end{aligned}
$$

where $h$ is the Fourier transform of the GW amplitude. An $h$-dependent term is also added to the equation relating $\mathbf{b}$ and $\mathbf{d}$ using $\mathbf{D}_{d}^{*}$ in place of $\mathbf{D}_{a}^{*}$. The parameter $\eta$ takes values from -1 to 1 depending on the orientation of the cavity and the polarization state of the incoming GW. For example, for a linearly polarized incoming GW, and for an optimally aligned Michelson interferometer, we have $\eta=1$ for one and -1 for the other.

It is straightforward to incorporate Eq. (54) into the general equation Eq. (2). In particular, the term containing $h$ on RHS contributes to the GW part of the general input field $\mathbf{u}$, i.e., to the third term of Eq. (3), with

$$
\mathbf{H}=-\eta \frac{\omega L}{2 c \sqrt{\hbar \omega}} \mathbf{D}_{c}^{*}
$$

\section{B. Photodetection: signal and noise}

For our purposes, the photodetector serves two roles: first, it represents an open port, from which vacuum fluctuations enter the interferometer; second, it determines the measurement point. For the former, the input-output relation of a photodetector, as it contributes to the matrix $\mathcal{M}$ and the generalized input vector $\mathbf{u}^{(i)}$, is trivial and has been discussed in Sec. IIB Here we focus on the latter. At zero frequency, there is only contribution to $\mathbf{b}$ from the carrier laser, while at non-zero sideband frequencies, the detected fields at a photodetector comprise three components: the gravitational-wave signal, classical laser noise, and noise due to vacuum fluctuations in the detected mode. The outgoing field being detected, $\mathbf{b}$, has the general form [see Eqs. (2) and (3)]:

$$
\begin{aligned}
\mathbf{b} & =\sum_{i}\left[\mathcal{M}^{-1}\right]_{b i}\left[\mathbf{v}^{(i)}+\mathbf{l}^{(i)}+\mathbf{H}^{(i)} h\right] \\
& \equiv \sum_{i} \mathcal{T}_{b i}\left[\mathbf{v}^{(i)}+\mathbf{l}^{(i)}+\mathbf{H}^{(i)} h\right]
\end{aligned}
$$

The summation is performed over all fields. We note that contributions to $\mathbf{v}^{(i)}$ exist only for fields that emerge from beam blocks or lossy optical elements, those to $\mathbf{l}^{(i)}$ exist only for the field that emerges from the laser, and those to $\mathbf{H}^{(i)}$ only for fields that emerge from cavities.

We suppose homodyne detection at quadrature angle, $\zeta$, is performed such that the measured field is

$$
\mathbf{b}_{\zeta}=b_{1} \cos \zeta+b_{2} \sin \zeta
$$

For a complete simulation, $\zeta$ should be the phase of the carrier that emerges at this port. However, in theoretical studies, we could also assign another value to $\zeta$, assuming that the local-oscillator phase is modified by some other means the simulation does not address.

For the detected field, the quantum noise spectral density is (see, e.g., Sec. III of Ref. [4])

$$
\left(N_{Q}^{2}\right)_{b}=\sum_{i}\left[\begin{array}{ll}
\cos \zeta & \sin \zeta
\end{array}\right] \mathcal{T}_{b i} S_{\mathbf{v}_{i}} \mathcal{T}_{b i}^{\dagger}\left[\begin{array}{c}
\cos \zeta \\
\sin \zeta
\end{array}\right]
$$

Because $\mathbf{v}_{i}$ is always proportional to a vacuum field, we have used $S_{\mathbf{v}_{i}}$ to denote the noise spectral density which is identical for all its quadratures. Here we have added the power of different loss contributions, since we assume the vacuum fields to be independent to each other. In general, laser noise is neither quantum-limited, nor are the magnitudes of phase and amplitude fluctuations equal; there could also be correlations between the laser amplitude and phase noise, even as the laser field enters the system. Taking these into account, we have a laser noise spectral density of

$$
\left(N_{L}^{2}\right)_{b}=\left[\begin{array}{ll}
\cos \zeta & \sin \zeta
\end{array}\right] \mathcal{T}_{b l} \mathcal{S}_{L} \mathcal{T}_{b l}^{\dagger}\left[\begin{array}{c}
\cos \zeta \\
\sin \zeta
\end{array}\right]
$$

where $l$ corresponds to the input laser field, and

$$
\mathcal{S}_{L} \equiv\left[\begin{array}{ll}
S_{11} & S_{12} \\
S_{12} & S_{22}
\end{array}\right]
$$

describes noise of the laser as it first enters the interferometer, with $S_{11(22)}$ being the noise spectral density of the first (second) quadrature, and $S_{12}$ the cross spectral density between the two quadratures. [In the usual convention of having input laser in the first quadrature, 1 corresponds to the amplitude quadrature, hence amplitude noise, while 2 corresponds to the phase quadrature, 
hence phase noise.] The transfer function for the GW signal is

$$
H_{b} \equiv \sum_{i}[\cos \zeta \sin \zeta] \mathcal{T}_{b i} \mathbf{H}^{(i)} .
$$

Note that GW contributions from different parts of the system add up coherently. The displacement (strain) noise spectral density from quantum noise is then given by

$$
S_{h}=\frac{N_{Q}^{2}+N_{L}^{2}}{|H|^{2}}
$$

\section{APPLICATION TO A COMPLEX INTERFEROMETER}

The mathematical formulation described in Sections II through $\mathrm{V}$ was encoded into a simulation program written in $\mathrm{C}++$. In this section we describe tests of the simulation code for a complex interferometer configuration, where the simulation results were compared with analytic calculations.

The interferometer configuration is shown in Fig. [5 and in Fig. [6] we show fields propagating in the interferometer as well as modes of motion of the mirrors. The interferometer is similar to that used in GW detection: a Michelson interferometer with Fabry-Perot cavities in each arm. All the mirrors of the interferometer are suspended as pendulums. Power-recycling [12] is optional and is not included here. The configuration shown has a few unusual features compared with a conventional interferometer, however. First, the end mirrors of the arm cavities are a common suspended object, coated with a high-reflectivity coating on both surfaces and assumed to have an opaque substrate. Second, this cavity end mirror object is very light, with a typical mass of $1 \mathrm{~g}$, and is suspended as a pendulum with resonant frequency of

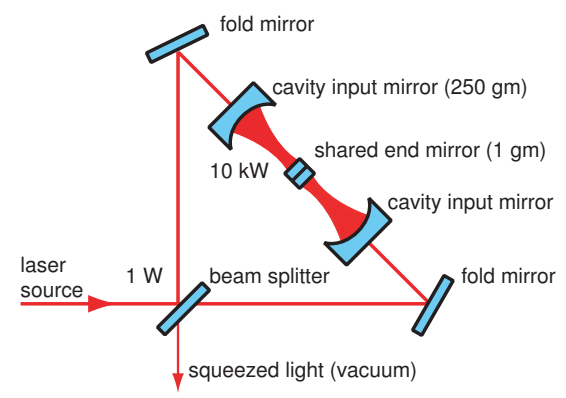

FIG. 5: Schematic of a an interferometer designed to extract ponderomotively squeezed light due to radiation-pressureinduced motion of the ultra-light shared mirror. Light from a highly intensity- and frequency-stabilized laser source is incident on the beamsplitter. High-finesse Fabry-Perot cavities in the arms of the Michelson interferometer are used to build up the carrier field incident on the end mirrors of the cavity, which are a single mechanical object. about $1 \mathrm{~Hz}$. All remaining optics are assumed to have a mass of $250 \mathrm{~g}$, and are also suspended as pendulums with a resonant frequency of $1 \mathrm{~Hz}$. Third, the cavities are detuned from resonance.

Testing the simulation with this somewhat unconventional interferometer configuration served two purposes: (i) It is the baseline design for an experiment to generate squeezed states of the electromagnetic field, produced with radiation-pressure-induced optical forces in an interferometer with low-mass mirror oscillators and high stored power [13]; and (ii) the shared end mirror gives rise to unexpected dynamical effects that prove interesting and instructive to explore, and are relevant to other high-power interferometers, such as Advanced LIGO [1]. We note that the shared end mirror has advantages in terms of mechanical stability and control system design, but the desired radiation-pressure effects can be realized by a configuration with two independent end mirrors as well.

\begin{tabular}{lcll}
\hline Parameter & Symbol & Value & Units \\
\hline \hline Light wavelength & $\lambda_{0}$ & 1064 & $\mathrm{~nm}$ \\
End mirror mass & $m$ & 1 & $\mathrm{~g}$ \\
Input mirror mass & $M$ & 0.25 & $\mathrm{~kg}$ \\
Input mirror transmission & $T_{i}$ & $4 \times 10^{-4}$ & - \\
Arm cavity finesse & $\mathcal{F}$ & $1.6 \times 10^{4}$ & - \\
Loss per bounce & - & $5 \times 10^{-6}$ & - \\
Arm cavity detuning & $\phi$ & $10^{-5}$ & $\lambda_{0}$ \\
Input power & $I_{0}$ & 1 & $\mathrm{~W}$ \\
\hline BS reflectivity asymmetry & $\Delta_{\mathrm{BS}}$ & 0.01 & - \\
Michelson phase imbalance & $\Delta_{\mathrm{M}}$ & \\
Michelson loss imbalance & $\Delta_{\mathrm{M}}$ & \\
Input mirror mismatch & $\Delta_{T}$ & $5 \times 10^{-6}$ & - \\
Detuning mismatch & $\Delta_{\phi}$ & $10^{-7}$ & $\lambda_{0}$ \\
Arm cavity loss mismatch & $\Delta_{\epsilon}$ & $2 \times 10^{-6}$ & - \\
\hline \hline
\end{tabular}

TABLE I: Select interferometer parameters and their nominal values.

\begin{tabular}{ccc}
\hline \hline$\epsilon$ & bandwidth & $\left(T_{i}+T_{e}\right) c /(4 L)$ \\
$\epsilon_{\mathrm{L}}$ & bandwidth due to loss & $T_{e} c /(4 L)$ \\
$-\lambda$ & resonant frequency & $\phi c / L$ \\
$\alpha$ & characteristic quadrature rotation angle & $\arctan (\lambda / \epsilon)$ \\
\hline \hline
\end{tabular}

TABLE II: Quantities associated with the detuned arm cavities.

\section{A. Ideal optical springs}

In this section we study analytically a crucial component of the interferometer design: the optical spring effect, especially in the case of two identical detuned cavities with a common end mirror. The input-output 


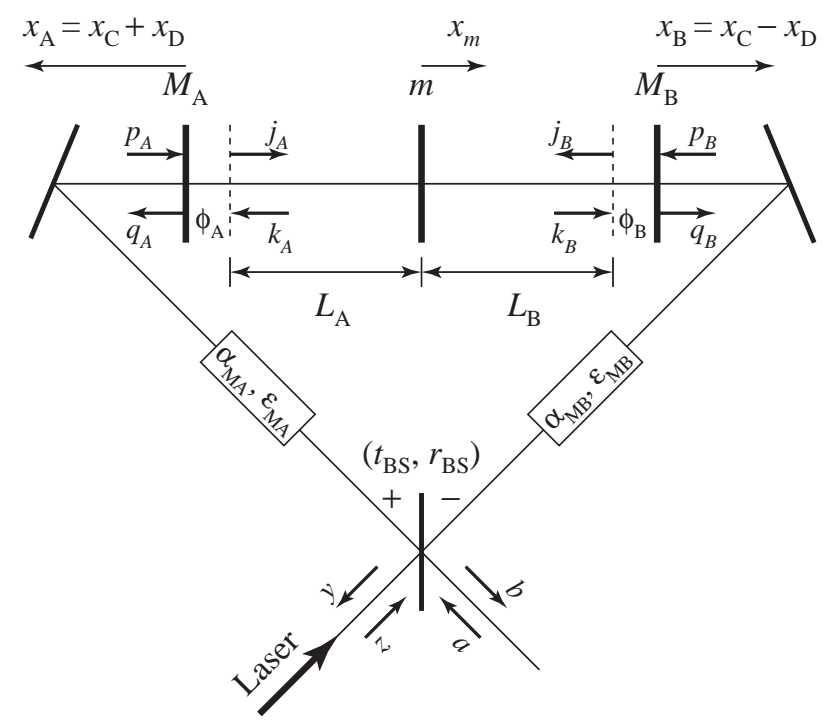

FIG. 6: Optical fields propagating in the interferometer, and modes of motion of the mirrors. In particular, $\alpha_{\mathrm{MA}, \mathrm{B}}$ and $\epsilon_{\mathrm{MA}, \mathrm{B}}$ are artificial detunings and losses one can add to the two arms of the Michelson interferometer, respectively, see Sec. VIB for their significance.

relation of this system can be obtained by carrying out our generic procedure analytically. In doing so, we extend previous results in Refs. [5, 14 to include two new features. First, we consider motions of all three mirrors, with mass of the input mirrors different from that of the common end mirror. Second, in our system the carrier phases incident on mirrors are different; under such a cir- cumstance, formulas developed in Sec. $\square$ are non-trivial extensions to existing ones.

In order to make results intuitively understandable, we consider only the ideal system, with the two input mirrors completely identical, the common mirror perfectly reflective on both sides, the two cavities having exactly the same lengths, the carrier incident on both input mirrors having equal amplitude and phase, and with a perfect beamsplitter. We also ignore the free pendulum frequency, and consider the test masses to be free. Similar to previous studies, we assume a high-finesse cavity and ignore the interaction between the motion of the input mirror and the carrier light outside the cavity. We retain terms only to the leading order in $\epsilon L / c, \lambda L / c$ and $\Omega L / c$, where $L$ is the cavity length, $c$ is the speed of light, $\Omega$ is the sideband frequency, and $(-\lambda-i \epsilon)$ is the complex optical resonant frequency of the cavity with fixed mirrors $[-\lambda$ denotes the resonant frequency and $\epsilon$ the bandwidth, defined in Table 1 and we ignore end-mirror loss].

\section{Differential Mode}

With the above assumptions, the differential optical mode couples only to differential modes of mirror motion: those with the two input masses moving such that $x_{A}=$ $-x_{B} \equiv x_{\mathrm{D}}$, and arbitrary $x_{m}$ [see Fig. [6] such modes form a two-dimensional subspace of all possible motions of the three mirrors. In the ideal case, we only need to study this mode. The differential input-output relation is given by

$$
\left(\begin{array}{l}
b_{1} \\
b_{2}
\end{array}\right)=\frac{1}{\mathcal{M}_{\mathrm{D}}} \mathbf{R}_{\alpha}\left[\mathbf{C}_{\mathrm{D}} \mathbf{R}_{-\alpha}\left(\begin{array}{l}
a_{1} \\
a_{2}
\end{array}\right)+\mathbf{s}_{\mathrm{D}}\left[x_{m}^{(0)}+x_{\mathrm{D}}^{(0)}\right]\right],
$$

with

$$
\mathbf{C}_{\mathrm{D}}=\left[\begin{array}{cc}
-\left(\Omega^{2}-\lambda^{2}+\epsilon^{2}\right) \Omega^{2}-\lambda \iota_{\mathrm{D}} & 2 \epsilon \lambda \Omega^{2} \\
-2 \epsilon \lambda \Omega^{2}+2 \epsilon \iota_{\mathrm{D}} & -\left(\Omega^{2}-\lambda^{2}+\epsilon^{2}\right) \Omega^{2}-\lambda \iota_{\mathrm{D}}
\end{array}\right], \quad \mathbf{s}_{\mathrm{D}}=\frac{2 \sqrt{\epsilon \iota_{\mathrm{D}} \Omega^{2}}}{L h_{\mathrm{SQL}}^{\mathrm{D}}}\left(\begin{array}{c}
\lambda \\
-\epsilon+i \Omega
\end{array}\right)
$$

and

$$
\mathcal{M}_{\mathrm{D}}=\Omega^{2}\left[(\Omega+i \epsilon)^{2}-\lambda^{2}\right]+\lambda \iota_{\mathrm{D}} .
$$

Here $x_{m}^{(0)}$ is the motion of a free end mirror with the same mass, $x_{\mathrm{D}}^{(0)}$ is the free differential motion of the input mirrors $\left(x_{A}^{(0)}=-x_{B}^{(0)}=x_{\mathrm{D}}^{(0)}\right) ; \alpha=\arctan (\lambda / \epsilon)$ is the carrier phase at the end mirror. The carrier incident on the input mirrors has phase 0 , the carrier inside the cavity, leaving the input mirror has phase $\alpha-\phi$, while the carrier inside the cavity entering the input mirror has phase $\alpha+\phi$. The quantity $h_{\mathrm{SQL}}^{\mathrm{D}}$ is the free-mass
Standard Quantum Limit associated with the differential mode, given by

$$
h_{\mathrm{SQL}}^{\mathrm{D}}=\sqrt{\frac{2 \hbar}{\mu_{\mathrm{D}} \Omega^{2} L^{2}}}, \quad \mu_{\mathrm{D}} \equiv 2 m M /(m+2 M) .
$$

The quantity $\iota_{\mathrm{D}}$, defined by

$$
\iota_{\mathrm{D}}=\frac{8 \omega_{0} I_{c}}{\mu_{\mathrm{D}} L c},
$$

measures the strength of optomechanical coupling [notice the dependence on carrier intensity $I_{c}$ and the inverse de- 
pendence on the effective mass of the differential mode mechanical oscillator $\mu_{\mathrm{D}}$ ]. Roots of $\mathcal{M}_{\mathrm{D}}$ are the (complex) resonant frequencies of the coupled optomechanical system. From $\iota_{\mathrm{D}}$ we define a characteristic frequency,

$$
\Theta_{\mathrm{D}} \equiv \sqrt{\iota_{\mathrm{D}} \lambda /\left(\epsilon^{2}+\lambda^{2}\right)} .
$$

For systems with $\Theta_{\mathrm{D}} \ll \epsilon$, the two resonances are well separated, and are given approximately by $\pm \Theta_{\mathrm{D}}$ [mechanical frequency due to optical spring] and $( \pm \lambda-i \epsilon)$ [optical resonant frequency], respectively - this is indeed the regime in which we construct our experiment.

The differential optical mode couples to a twodimensional subspace of all possible motions of the three mirrors. It is instructive to look at the motion of separate mirrors, in the regime of $\Omega \ll \epsilon$, i.e. for sideband frequencies $\Omega$ well within the linewidth of the cavities:

$$
\begin{aligned}
{\left[\begin{array}{c}
x_{m} \\
x_{\mathrm{D}}
\end{array}\right]=} & \frac{1}{\Theta_{\mathrm{D}}^{2}-\Omega^{2}} \\
& {\left[\begin{array}{cc}
\frac{\Theta_{\mathrm{D}}^{2}}{\Lambda^{2}+1}-\Omega^{2} & -\frac{\Lambda^{2} \Theta_{\mathrm{D}}^{2}}{\Lambda^{2}+1} \\
-\frac{\Theta_{\mathrm{D}}^{2}}{\Lambda^{2}+1} & \frac{\Lambda^{2} \Theta_{\mathrm{D}}^{2}}{\Lambda^{2}+1}-\Omega^{2}
\end{array}\right]\left[\begin{array}{c}
x_{m}^{(0)} \\
x_{\mathrm{D}}^{(0)}
\end{array}\right] . }
\end{aligned}
$$

Here we have defined $\Lambda^{2} \equiv 2 M / m$. From Eq. [69], we conclude immediately that

$$
x_{m}+x_{\mathrm{D}}=-\frac{\Omega^{2}}{\Theta_{\mathrm{D}}^{2}-\Omega^{2}}\left[x_{m}^{(0)}+x_{\mathrm{D}}^{(0)}\right] .
$$

This change in response is exactly what happens when a free test particle is connected to a spring with mechanical resonant frequency $\Theta_{D}$. Equation (70) reveals a crucial advantage of the optical spring - that the response of the cavity length to external disturbances (driven by seismic and/or thermal forces, e.g.) is greatly suppressed from the corresponding value for free-mass systems. Theoretically, this suppression is present even when a mechanical spring is used. However, mechanical springs introduce thermal noise, usually of much higher magnitude due to the intrinsic mechanical loss [14, 15].

It is interesting to notice that the suppression of total cavity length fluctuations is achieved collectively by the end mirror and the input mirror. As we see from Eq. (69), [in the case of large $\Lambda$ ], the motion of the end mirrors $x_{\mathrm{m}}$ is suppressed from its free mass value by the factor in Eq. (70), while the motion of the input mirrors $x_{\mathrm{D}}$ is not influenced by the spring, since it is relatively massive. Fortunately, through the $(1,2)$ component of the matrix on the RHS of Eq. 69), this motion of the input mirror is imposed onto the end mirror with opposite sign, again suppressing the total cavity length fluctuations.

Now let us restrict ourselves to the regime of $\Omega<\Theta_{D}<$ $\epsilon$, and study the quantum fluctuations and classical component of the output field (due to classical disturbances to the mirrors). As we shall see shortly, this regime has two crucial features: (i) the response of the output field to $x_{m}^{(0)}+x_{\mathrm{D}}^{(0)}$, and thus length fluctuations due to seismic and thermal noise, are greatly suppressed by the optical spring and (ii) the output squeezed state is frequencyindependent.

For quantum fluctuations, we have

$$
\frac{\mathbf{C}_{\mathrm{D}}}{\mathcal{M}_{\mathrm{D}}} \rightarrow\left[\begin{array}{cc}
-1 & 0 \\
2 \epsilon / \lambda & -1
\end{array}\right],
$$

which is frequency-independent. It is straightforward to derive that the quantum noise spectrum in the $b_{\zeta} \equiv$ $b_{1} \cos \zeta+b_{2} \sin \zeta$ quadrature [Cf. Eq. [58]):

$$
S_{\zeta} \rightarrow 1+\frac{2 \epsilon^{2}}{\lambda^{2}}-2 \sqrt{\frac{\epsilon^{2}}{\lambda^{2}}+\frac{\epsilon^{4}}{\lambda^{4}}} \cos (2 \zeta-3 \alpha) .
$$

In particular, terms in $\epsilon / \lambda$ are associated with squeezing, where the constant power squeeze factor $e^{2 q}(q>0)$ is given by

$$
\sinh q=|\epsilon / \lambda| .
$$

The minimum noise spectral density $\left(S_{\zeta}=e^{-2 q}\right)$ is reached at $\zeta=3 \alpha / 2$, while at $\zeta=\alpha$ and $2 \alpha$ the noise spectrum is equal to the vacuum level $\left(S_{\zeta}=1\right)$. Values of $\epsilon / \lambda$ corresponding to several power squeeze factors are listed in Table III As shown, $\epsilon$ and $\lambda$ will not differ by a factor of more than $\sim 2$, for typically desired squeeze factors.

Now for the classical component, given by the second term in Eq. [63), we have

$$
\frac{1}{\mathcal{M}_{\mathrm{D}}} \mathbf{R}_{\alpha} \mathbf{s}_{\mathrm{D}} \rightarrow \frac{2}{L h_{\mathrm{SQL}}^{\mathrm{D}}} \sqrt{\frac{\Omega^{2}}{\Theta_{\mathrm{D}}^{2}} \frac{\epsilon}{\lambda}}\left[\begin{array}{c}
\sin 2 \alpha \\
-\cos 2 \alpha
\end{array}\right] .
$$

This means the entire signal due to differential displacement $x_{m}^{(0)}+x_{\mathrm{D}}^{(0)}$ is in the single quadrature $\zeta=2 \alpha+\pi / 2$, and there is no $x_{m}^{(0)}+x_{\mathrm{D}}^{(0)}$ signal in the $\zeta=2 \alpha$ quadrature. Interestingly, the quantum noise in this quadrature is right at vacuum level. In addition, since $h_{\mathrm{SQL}}^{\mathrm{D}} \propto 1 / \Omega$, the response of $b_{\zeta}$ to $x_{m}^{(0)}+x_{\mathrm{D}}^{(0)}$ is proportional to $\Omega^{2}$ at this regime - therefore not only the motion, but also the output field, has a suppressed response to thermal and seismic noises. Note here that the suppression factor is proportional to $\sqrt{I_{c}}$ (since $\theta_{\mathrm{D}} \propto \sqrt{\iota_{\mathrm{D}}} \propto \sqrt{I_{c}}$ ) - because motion is suppressed by $I_{c}$, while the optical sensing of mirror motion is enhanced by $\sqrt{I_{c}}$. Now suppose we introduce a noisy force which induces a spectral density $S_{x}^{N}$ on a free mass, then the output classical noise will be

$$
S_{\zeta}^{N}=4 \frac{\Omega^{2}}{\Theta_{D}^{2}} \frac{\epsilon}{\lambda} \sin ^{2}(\zeta-2 \alpha) \frac{S_{x}}{L^{2}\left(h_{\mathrm{SQL}}^{\mathrm{D}}\right)^{2}} .
$$

At the minimum quantum noise quadrature, $\zeta=3 \alpha / 2$, we have

$$
\begin{aligned}
S_{3 \alpha / 2}^{N} & =\frac{2 \epsilon}{\lambda}\left[1-\frac{\epsilon}{\sqrt{\lambda^{2}+\epsilon^{2}}}\right] \frac{\Omega^{2}}{\Theta_{D}^{2}} \frac{S_{x}}{L^{2}\left(h_{\mathrm{SQL}}^{\mathrm{D}}\right)^{2}} \\
& \leq 0.6 \frac{\Omega^{2}}{\Theta_{D}^{2}} \frac{S_{x}}{L^{2}\left(h_{\mathrm{SQL}}^{\mathrm{D}}\right)^{2}},
\end{aligned}
$$


where the inequality is obtained by taking maximum over all $\epsilon$ and $\lambda$. We note that because of the suppression factor $\Omega^{2} / \Theta^{2}$, the classical noise $S_{x}^{N}$ can be much higher than the free-mass Standard Quantum Limit while still allowing the interferometer to generate squeezed vacuum!

\begin{tabular}{|c|cccc|}
\hline Squeeze Factor $(\mathrm{dB})$ & 3 & 7 & 10 & 20 \\
\hline$\epsilon / \lambda$ & 0.58 & 1.13 & 1.42 & 2.12 \\
\hline
\end{tabular}

TABLE III: Relationship between power squeeze factor and $\epsilon / \lambda$, see Eq. [73).

\section{Common Mode}

We now consider the common optical mode, which couples with motion of the input mirrors corresponding to
$x_{A}=x_{B} \equiv x_{\mathrm{C}}$. This mode is irrelevant to an ideal interferometer with identical arms and perfect contrast. In reality, however, the common mode will influence the output via couplings induced by differences (mismatch) between the two cavities, for example. Such effects can be quite important near the common-mode optomechanical resonance.

The input-output relation of the common mode, similar to that of the differential mode [cf. Eq. [63)], is given by:

$$
\left(\begin{array}{l}
y_{1} \\
y_{2}
\end{array}\right)=\frac{1}{\mathcal{M}_{\mathrm{C}}} \mathbf{R}_{\alpha}\left[\mathbf{C}_{\mathrm{C}} \mathbf{R}_{-\alpha}\left(\begin{array}{c}
z_{1} \\
z_{2}
\end{array}\right)+\mathbf{s}_{\mathrm{C}} x_{\mathrm{C}}^{(0)}\right]
$$

with [cf. Eq. (64) ]

$$
\mathbf{C}_{\mathrm{C}}=\left[\begin{array}{cc}
-\left(\Omega^{2}-\lambda^{2}+\epsilon^{2}\right) \Omega^{2}-\lambda \iota_{\mathrm{C}} & 2 \epsilon \lambda \Omega^{2} \\
-2 \epsilon \lambda \Omega^{2}+2 \epsilon \iota_{\mathrm{C}} & -\left(\Omega^{2}-\lambda^{2}+\epsilon^{2}\right) \Omega^{2}-\lambda \iota_{\mathrm{C}}
\end{array}\right], \quad \mathbf{s}_{\mathrm{C}}=\frac{2 \sqrt{\epsilon \iota_{\mathrm{C}} \Omega^{2}}}{L h_{\mathrm{SQL}}^{\mathrm{C}}}\left(\begin{array}{c}
\lambda \\
-\epsilon+i \Omega
\end{array}\right)
$$

and [cf. Eq. [65)]

$$
\mathcal{M}_{\mathrm{C}}=\Omega^{2}\left[(\Omega+i \epsilon)^{2}-\lambda^{2}\right]+\lambda \iota_{\mathrm{C}} .
$$

$h_{\mathrm{SQL}}^{\mathrm{C}}$, the SQL associated with the common mode, is given by [cf. Eq. [66] ]

$$
h_{\mathrm{SQL}}^{\mathrm{C}}=\sqrt{\frac{2 \hbar}{\mu_{\mathrm{D}} \Omega^{2} L^{2}}}, \quad \mu_{\mathrm{C}}=2 M .
$$

The quantity $\iota_{\mathrm{C}}$ is given by [cf. Eq. [67)]

$$
\iota_{\mathrm{C}}=\frac{8 \omega_{0} I_{c}}{\mu_{\mathrm{C}} L c} .
$$

For the common mode, we have a optomechanical resonant frequency of [cf. Eq. [68)]

$$
\Theta_{\mathrm{C}} \equiv \sqrt{\iota_{\mathrm{C}} \lambda /\left(\epsilon^{2}+\lambda^{2}\right)}, \quad \text { if } \Theta_{\mathrm{C}} \ll \epsilon .
$$

This frequency is in general much lower than its differential-mode counterpart, with

$$
\frac{\Theta_{\mathrm{C}}}{\Theta_{\mathrm{D}}}=\sqrt{\frac{\iota_{\mathrm{C}}}{\iota_{\mathrm{D}}}}=\sqrt{\frac{\mu_{\mathrm{D}}}{\mu_{\mathrm{C}}}}=\sqrt{\frac{m}{m+2 M}} .
$$

\section{B. Laser coupling to the antisymmetric port due to mismatch}

Mismatch between the optical parameters of the two arm cavities, as well as imbalance in the beamsplitter reflection/transmission ratio and imperfect contrast of the

\begin{tabular}{c|cc}
\hline \hline$\Delta_{(k)}$ & $C_{(k)}$ & $\varphi_{(k)}^{\mathrm{C}}$ \\
\hline$\frac{\Delta \epsilon}{\epsilon}$ & $-\frac{\epsilon \lambda}{\epsilon^{2}+\lambda^{2}}$ & $2 \alpha+\pi / 2$ \\
$\frac{\Delta \epsilon_{\mathrm{L}}}{\epsilon}$ & $-\frac{\epsilon}{\sqrt{\epsilon^{2}+\lambda^{2}}}$ & $\alpha$ \\
$\frac{\Delta \lambda}{\lambda}$ & $\frac{\epsilon \lambda}{\epsilon^{2}+\lambda^{2}}$ & $2 \alpha+\pi / 2$ \\
$\Delta \alpha_{\mathrm{M}}$ & 1 & $2 \alpha+\pi / 2$ \\
$\Delta_{\mathrm{BS}}$ & 0 & \\
$\Delta \epsilon_{\mathrm{M}}$ & $-\frac{1}{2}$ & $2 \alpha$ \\
\hline \hline
\end{tabular}

TABLE IV: Transfer function from carrier to differential output [see Eq. 91]], in the leading-order approximation. The same coefficients apply to phase-noise coupling, i.e., $N_{k}^{\mathrm{P}}=C_{(k)}, \varphi_{k}^{\mathrm{P}}=\varphi_{(k)}^{\mathrm{C}}$, in the low-frequency regime [see Eq. [95]].

Michelson interferometer, can couple the carrier and also the noise sidebands on the laser to the differential detection port. For each arm, A and B, we denote the true value of the $k$ th quantity by its nominal value plus contributions due to imperfections, i.e.

$$
X_{(k) A, B}=X_{(k)} \pm \frac{1}{2} \Delta X_{(k)} .
$$

Here the index $k$ refers to the type of imperfection being considered. The beamsplitter asymmetry is character- 


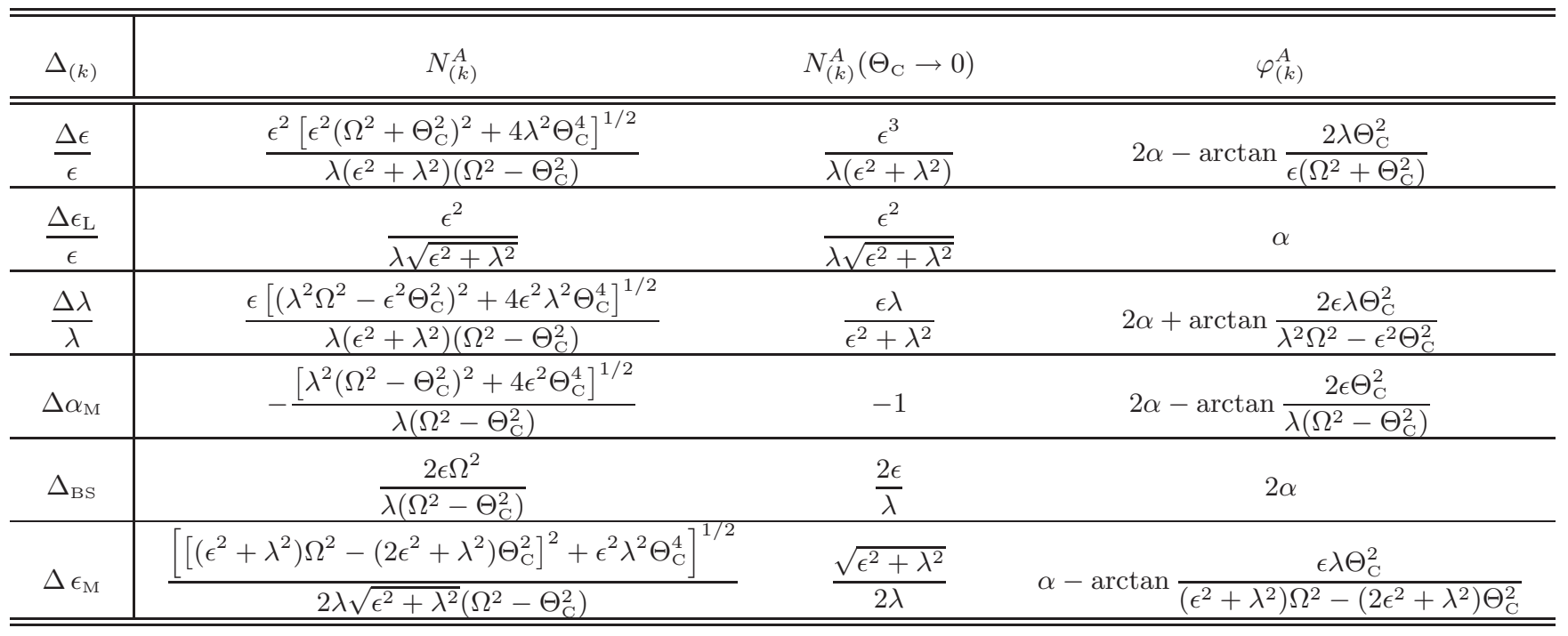

TABLE V: Laser amplitude noise coupling into the dark port, in the leading-order approximation and low-frequency regime [see Eq. 95].

ized by

$$
\Delta_{\mathrm{BS}}=t_{\mathrm{BS}}^{2}-r_{\mathrm{BS}}^{2}
$$

Michelson imperfections can be characterized by the difference in the phase shifts and losses when light travels from the beamsplitter to the input mirrors of the two arms:

$$
\alpha_{\mathrm{MA}, \mathrm{B}}=\alpha_{\mathrm{M}} \pm \frac{1}{2} \Delta \alpha_{\mathrm{M}}, \quad \epsilon_{\mathrm{MA}, \mathrm{B}}=\epsilon_{\mathrm{M}} \pm \frac{1}{2} \Delta \epsilon_{\mathrm{M}} .
$$

In addition to $\Delta_{\mathrm{BS}}, \Delta \alpha_{\mathrm{M}}$ and $\Delta \epsilon_{\mathrm{M}}$, which concern the beamsplitter, we consider the following contributions to mismatch between the arms,

$$
\begin{aligned}
T_{i \mathrm{~A}, \mathrm{~B}} & \equiv T_{i} \pm \frac{1}{2} \Delta_{T} \\
T_{e \mathrm{~A}, \mathrm{~B}} & \equiv T_{e} \pm \frac{1}{2} \Delta_{\epsilon} \\
\phi_{\mathrm{A}, \mathrm{B}} & \equiv \phi \pm \frac{1}{2} \Delta_{\phi}
\end{aligned}
$$

that is, mismatch between input mirror power transmissivities, end mirror losses and cavity detuning, respectively. We replace these with the following more convenient quantities:

$$
\frac{\Delta \epsilon}{\epsilon}=\frac{\Delta_{T}}{T_{i}+T_{e}}, \quad \frac{\Delta \epsilon_{\mathrm{L}}}{\epsilon}=\frac{\Delta_{\epsilon}}{T_{i}+T_{e}}, \quad \frac{\Delta \lambda}{\lambda}=\frac{\Delta_{\phi}}{\phi} .
$$

[See Table I for definitions of $\epsilon, \epsilon_{\mathrm{L}}$ and $\lambda$.]

In the remainder of this section, we give the transfer functions from the carrier light (DC), laser amplitude fluctuations and laser phase fluctuations to the differential output port, to first order in the mismatch (recall that ideally, in the absence of imperfections, these common-mode inputs do not appear in the differential output port). We keep our formulae to the leading order in $\{\Omega L / c, \epsilon L / c, \lambda L / c\}$, and ignore the averaged losses, $\epsilon_{\mathrm{L}}$ and $\epsilon_{\mathrm{M}}$ (but not $\Delta \epsilon_{\mathrm{L}}$ and $\Delta \epsilon_{\mathrm{M}}$ ). We refer to this as the leading-order approximation. Furthermore, in order to keep the analytical results understandable, we work only in the regime of $\left\{\Omega, \Theta_{\mathrm{C}}\right\} \ll\left\{\Theta_{\mathrm{D}}, \lambda, \epsilon\right\}$, which we shall refer to as the low-frequency regime.

Definitions and assumed values for $\Delta_{\mathrm{BS}}, \Delta \alpha_{\mathrm{M}}, \Delta \epsilon_{\mathrm{M}}$, $\Delta_{\mathrm{T}}, \Delta_{\phi}$, and $\Delta_{\epsilon}$ are given in Table

\section{The Carrier}

The transfer function from the carrier to the differential output can be written as

$$
\sum_{k} \Delta_{(k)} C_{(k)}\left(\begin{array}{c}
\cos \varphi_{(k)}^{\mathrm{C}} \\
\sin \varphi_{(k)}^{\mathrm{C}}
\end{array}\right)
$$

where definitions of $\Delta_{(k)}$, values of $C_{(k)}$ and $\varphi_{(k)}^{\mathrm{C}}$ are listed in Table IV assuming the carrier at the beamsplitter is in the first (amplitude) quadrature.

Contributions listed in Table IV can all be obtained from simple considerations. First, since each field that interferes at the beamsplitter is scaled by one transmission and one reflection coefficient factor, $\Delta_{\mathrm{BS}}$ does not contribute to the output carrier light at the differential port. Then, for all mismatches except the loss, one only has to notice that when the arm cavities are lossless, carrier light with amplitude $D$ and phase $\varphi=0$ returns to the beamsplitter with amplitude reduced to $\left(1-\epsilon_{\mathrm{M}}\right)$, and quadrature rotated by $2 \alpha+2 \alpha_{\mathrm{M}}$. As a consequence, the differential output port gets $(D / 2)\left(-\Delta \epsilon_{\mathrm{M}}\right)=\left(-\Delta \epsilon_{\mathrm{M}} / 2\right) D$ in the $\varphi=2 \alpha$ quadrature (factor of 2 due to the beamsplitter), 
and

$$
\begin{aligned}
& (D / 2) \Delta\left[2 \alpha+2 \alpha_{\mathrm{M}}\right] \\
= & {\left[\frac{\epsilon \lambda}{\epsilon^{2}+\lambda^{2}}\left(-\frac{\Delta \epsilon}{\epsilon}+\frac{\Delta \lambda}{\lambda}\right)+\Delta \alpha_{\mathrm{M}}\right] D }
\end{aligned}
$$

in the orthogonal quadrature, $\varphi=2 \alpha+\pi / 2$. The effect of the loss mismatch can be understood when we decompose the (complex) reflectivity of the cavity into a sum of two components:

$$
\begin{aligned}
\frac{\sqrt{R_{e}} e^{2 i \phi}-\sqrt{R_{i}}}{1-\sqrt{R_{i} R_{e}} e^{2 i \phi}} & =\frac{1+i \lambda / \epsilon}{1-i \lambda / \epsilon}-\frac{\epsilon_{\mathrm{L}}}{\epsilon} \frac{2}{1-i \lambda / \epsilon} \\
& =e^{2 i \alpha}-\frac{\epsilon_{\mathrm{L}}}{\epsilon} \frac{2 \epsilon}{\sqrt{\epsilon^{2}+\lambda^{2}}} e^{i \alpha}
\end{aligned}
$$

Here we see that the loss $\epsilon_{\mathrm{L}}$ creates an output at the $\varphi=$ $\alpha$ quadrature, so an imbalance in loss $\Delta \epsilon_{\mathrm{L}}$ will contribute

$$
\left(-\frac{\Delta \epsilon_{\mathrm{L}}}{\epsilon} \frac{\epsilon}{\sqrt{\epsilon^{2}+\lambda^{2}}}\right) D
$$

in the $\varphi=\alpha$ quadrature in the differential output port.

\section{Amplitude (Intensity) and Phase (frequency) Noise}

Under our simplifications, the laser amplitude noise $z_{1}$ and phase noise $z_{2}$ couple to single (yet frequency dependent) quadratures in the differential output port, as parametrized by

$$
\begin{aligned}
\sum_{k} \Delta_{(k)} & {\left[N_{(k)}^{\mathrm{A}}\left(\begin{array}{c}
-\sin \varphi_{(k)}^{\mathrm{A}} \\
\cos \varphi_{(k)}^{\mathrm{A}}
\end{array}\right) z_{1}\right.} \\
& \left.+N_{(k)}^{\mathrm{P}}\left(\begin{array}{c}
-\sin \varphi_{(k)}^{\mathrm{P}} \\
\cos \varphi_{(k)}^{\mathrm{P}}
\end{array}\right) z_{2}\right] .
\end{aligned}
$$

Measurement of the output quadrature $b_{\zeta} \equiv b_{1} \cos \zeta+$ $b_{2} \sin \zeta$ will include the laser noise:

$$
\sum_{(k)} \Delta_{(k)}\left[N_{(k)}^{\mathrm{A}} z_{1} \sin \left(\varphi_{(k)}^{\mathrm{A}}-\zeta\right)+N_{(k)}^{\mathrm{P}} z_{2} \sin \left(\varphi_{(k)}^{\mathrm{P}}-\zeta\right)\right] .
$$

In particular, the output quadrature $\zeta=\varphi_{(k)}^{\mathrm{A}(\mathrm{P})}$ is not sensitive to the $k$-th contribution of laser amplitude (phase) noise [note that we have switched the notation for $\varphi$ from that of Eq. [91]).

As it also turns out, in the leading-order approximation and the low-frequency regime, $\left[N_{(k)}^{\mathrm{P}}, \varphi_{(k)}^{\mathrm{P}}\right]=$ $\left[C_{(k)}, \varphi_{(k)}\right]$. Considering the different ways $\varphi$ appears in Eqs. (91) and 295), this means the phase noise coupled to the differential output port remains orthogonal to the carrier. This can be argued for easily: since phase modulations on the carrier do not drive mirror motion, the propagation of phase noise is not affected by the optical spring. Amplitude modulations, on the other hand, do drive mirror motion and therefore should couple to the differential port in a dramatically different way. We tabulate the quantities $N_{(k)}^{\mathrm{A}}$ and $\varphi_{(k)}^{\mathrm{A}}$ in Tab. $\mathrm{V}$ from which we can see that the amplitude-noise coupling has features around the common-mode optical-spring resonant frequency, $\Theta_{\mathrm{C}}$.

\section{Evading Laser Noise by Artificial Asymmetry}

For realistically achievable symmetry between the two arms, laser noises turn out to be the dominant noise source to our squeezer. Here we discuss a novel way of mitigating laser noise coupling by introducing artificial asymmetries. According to the approximate results (in the leading-order approximation and low-frequency regime) obtained in the previous section, both amplitude and phase noise emerge from single quadratures (as vector sums of contributions from different mechanisms). We can, therefore, eliminate the laser noise totally, up to this order, if we make both of them emerge from the same quadrature $\zeta+\pi / 2$, and make sure that the orthogonal quadrature, $\zeta$, has a sub-vacuum noise spectrum. At our disposal are two asymmetries that we can adjust manually: $\Delta \alpha_{\mathrm{M}}$ and $\Delta \epsilon_{\mathrm{M}}$.

At any given sideband frequency $\Omega$, for a generic set of other asymmetries, it is always possible to make both laser noise sources emerge at the $\zeta+\pi / 2$ quadrature (and, therefore, to vanish at the $\zeta$ quadrature), by adjusting $\Delta \alpha_{\mathrm{M}}$ and $\Delta \epsilon_{\mathrm{M}}$, if the following non-degeneracy condition is satisfied:

$$
\begin{aligned}
& \Delta_{\text {laser }}(\Omega, \zeta) \\
\equiv & \operatorname{det}\left[\begin{array}{ll}
\sin \left(\varphi_{\alpha_{\mathrm{M}}^{\mathrm{M}}}^{\mathrm{A}}-\zeta\right) N_{\Delta \alpha_{\mathrm{M}}}^{\mathrm{A}} & \sin \left(\varphi_{\Delta \epsilon_{\mathrm{M}}}^{\mathrm{A}}-\zeta\right) N_{\Delta \epsilon_{\mathrm{M}}}^{\mathrm{A}} \\
\sin \left(\varphi_{\alpha_{\mathrm{M}}}^{\mathrm{P}}-\zeta\right) N_{\Delta \alpha_{\mathrm{M}}}^{\mathrm{P}} & \sin \left(\varphi_{\Delta \epsilon_{\mathrm{M}}}^{\mathrm{P}}-\zeta\right) N_{\Delta \epsilon_{\mathrm{M}}}^{\mathrm{P}}
\end{array}\right] \\
\neq & 0 .
\end{aligned}
$$

[See Eq. 951).]

According to Tables IV and V laser phase noise emerges in a frequency-independent quadrature, but the amplitude noise does not. This means the elimination of laser noise must be frequency-dependent, and we can only choose one particular frequency for perfect laser noise evasion. However, if $\Omega \gg \Theta_{\mathrm{C}}$ is also satisfied, then the frequency-dependence goes away. We consider this special case, and choose a detection quadrature of $\zeta=3 \alpha / 2$, i.e., the one with minimum quantum noise. From Tabs. IV and $\nabla$ we get

$$
\left.\Delta_{\text {laser }}\left(\Omega, \frac{3}{2} \alpha\right)\right|_{\Theta_{\mathrm{C}} \rightarrow 0}=-\frac{\epsilon}{4 \sqrt{\epsilon^{2}+\lambda^{2}}} \neq 0 .
$$

Since the carrier always emerges $\pi / 2$ away from the phase noise, it emerges in exactly the same quadrature we propose to detect. In this way, the laser-noise-evading squeezer always produces squeezed light with amplitude squeezing. 
Finally, we note that, due to possible higher-order corrections, laser noise evasion may not be as perfect as predicted by our first-order approximation, even at a single frequency. The amount of residual laser noise, as well as the exact level of the deliberate asymmetries we introduce, must be given by a more accurate calculation.

\section{Comparison between analytical calculations and numerical simulations}

In Table \ we list the parameters used in modelling our interferometer. An important feature of the numerical code is that it can handle imperfections in the optics quite naturally, while for analytical techniques the solution becomes complicated rather dramatically when more ingredients are added. To fully test this feature, we constructed a test case with realistic imperfections. The imperfections included were those mentioned in Sec. VIB Using the parameters listed in Table I we calculate the noise at the differential port due to quantum fluctuations entering from this port and from lossy mirrors, as well as laser amplitude and phase fluctuations entering from the symmetric port.

In Fig. [7] we show the calculated noise levels from numerical simulations in curves, while those from the analytical treatment are shown as solid points. The agreement between the two sets of calculations is reassuring. Now we discuss these noise spectrum in more details. In the left panel of the Fig. [7 we plot noises due to vacuum fluctuations entering from the dark port (light curve and points), and due to vacuum fluctuations entering from mirror losses (dark curve and points). In both results, there is a rather dramatic resonant feature around the differential-mode optical-spring resonant frequency, at $\Theta_{\mathrm{D}} \approx 8 \mathrm{kHz}$, as can be expected from Sec. VIA The rather weak but still noticeable feature around the common-mode optical-spring resonant frequency $\Theta_{\mathrm{C}} \approx 360 \mathrm{~Hz}$ is solely due to optical parameter mismatch. In the right panel, we show laser amplitude (light curve and dots) and phase (dark curve and points) noises; we have introduced artificial asymmetries $\alpha_{\mathrm{M}}$ and $\epsilon_{\mathrm{M}}$, with values obtained empirically using the numerical simulation code, such that both laser noise sources are evaded to a roughly maximal extent at $1 \mathrm{kHz}$. For this reason, contributions to the results shown here are largely higher order, and we cannot hope to explain them using results obtained in Sec. VIB Here we do observe dramatic features around both the differential-mode and the common-mode optical-spring resonances.

Results in Fig. [7are also of great significance for a practical reason: they show that the vacuum modes exiting the interferometer are squeezed by a large factor even in the presence of realistic estimates for optical losses (left panel) and laser amplitude and phase noise (right panel).

\section{SUMMARY AND CONCLUSIONS}

The main purpose of this work was to develop a mathematical framework for the simulation of quantum fields in a complex interferometer that includes radiation pressure effects. We work in the linear regime around the operation point of this interferometer; in this regime, after adopting the Heisenberg picture of quantum mechanics, the quantum equations of motion (Heisenberg operators) of observables are identical to classical ones.

During the development of this framework, we augmented previous treatments of mirrors (and beamsplitters) by allowing the carrier phases at the four (eight) ports to be different. This extension gives rise to the optical spring effect even without detuned optical cavities.

Based on this mathematical framework, we developed a simulation code that can allow arbitrary optical topologies, and applied it to a specific example of the interferometer shown in Fig. 5] This interferometer was shown to be capable of squeezing the vacuum modes that enter - and subsequently exit - the differential port of the beamsplitter. We introduced optical spring effects by detuning the arm cavities as a means of mitigating the detrimental effects of thermal noise. We study not only the quantum noise, but also laser noise couplings from the symmetric (input or bright) port to the output (antisymmetric or dark) port. Good agreement was found between numerical results given by this code and analytical ones derived independently. This agreement makes us confident that the simulation is working correctly for this rather complex interferometer.

During our study of the laser noise couplings, we found a novel method of evading the laser noise by introducing artificial but controlled asymmetries. This is crucial for the practical implementation of this interferometer, and is likely to find applications in many other experiments.

Our simulation code is now being used in the detailed optical design of the Advanced LIGO interferometer. We also envisage the following extensions to the code in the near future:

- Allowing multiple carrier or rf sidebands, which may be relevant to the modeling of squeezing experiments that use nonlinear optical media, e.g., crystals, as well as the modeling of error signals for control systems.

- Incorporating the modeling of servo loops. Here we may rely on the input from quantum control theory as to whether and how realistically a Heisenberg treatment can describe a electro-optical feedback system.

- Allowing nonlinear media or other elements with "custom" dispersion relations. 

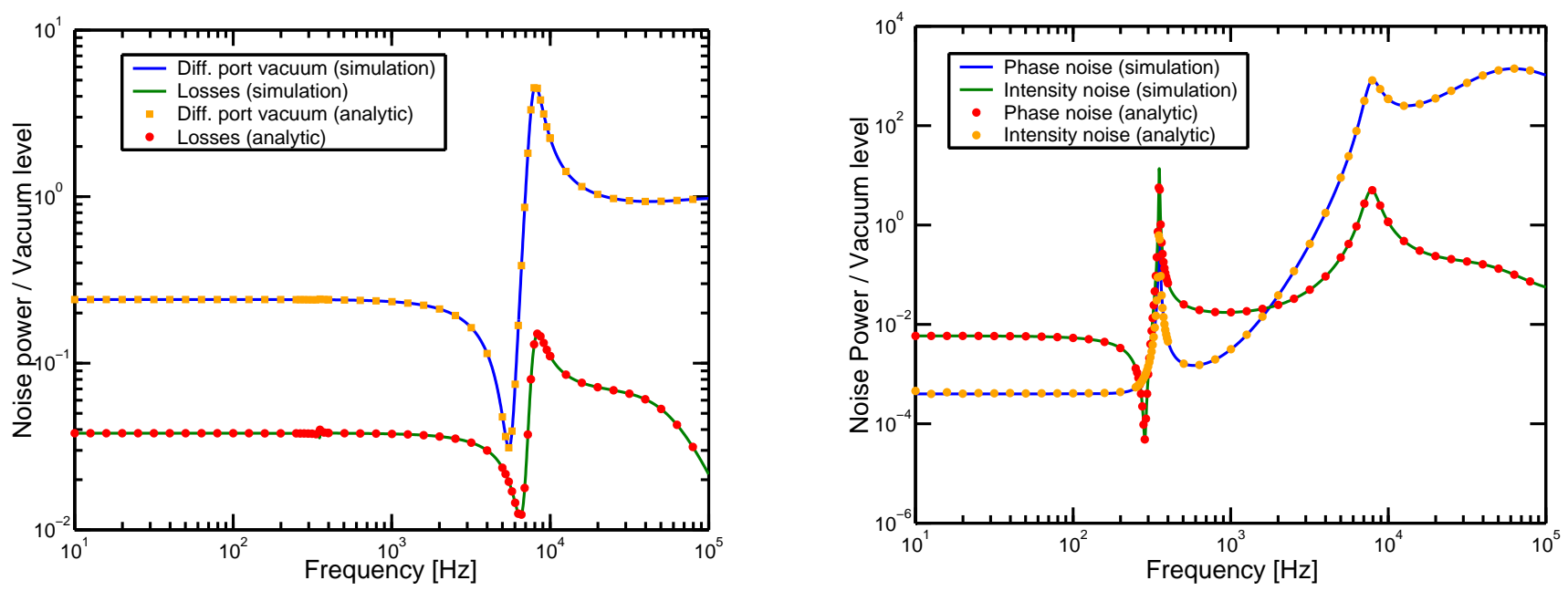

FIG. 7: Spectra of noise power at the output port of the ponderomotive interferometer, normalized to the vacuum noise level. The noise power is dimensionless, as compared to vacuum; a pure vacuum (or shot noise) corresponds to unity. The lines are results of the simulation code, while the data points are values calculated from the corresponding analytic calculations. In the left panel the vacuum noise level of the light exiting the antisymmetric port is shown. The solid (blue) curve shows the vacuum noise due to the (unsqueezed) vacuum fluctuations that enter via the antisymmetric port of the interferometer; the dashed (green) plot represents the noise due to the vacuum fluctuations that enter via other optical losses in the system. At all frequencies where the vacuum noise power is below unity, the vacuum modes exiting the interferometer are squeezed due to radiation-pressure effects. For the squeezing to be useful, all noise couplings must yield a lower noise power than the squeezed vacuum. In the right panel we show the coupling of laser frequency (solid, blue) and laser amplitude (dashed, green) noise fields to the output port, as calculated by the simulation code. Noise levels of $10^{-4} \mathrm{~Hz} / \mathrm{Hz}^{1 / 2}$ for frequency noise and $10^{-8} \mathrm{~Hz}^{-1 / 2}$ for amplitude noise are assumed at the input to the interferometer; all other parameters are listed in Table

\section{Acknowledgments}

We thank our colleagues at the LIGO Laboratory, especially Keisuke Goda and David Ottaway, for stimulating discussions. We gratefully acknowledge support from National Science Foundation grants PHY-0107417, PHY0300345 and (for Y.C.) PHY-0099568. Y.C.'s research was also supported by the David and Barbara Groce Fund at the San Diego Foundation, as well as the Sofja Kovalevskaja Programme (funded by the German Federal Ministry of Education and Research). Y.C. thanks the MIT LIGO Laboratory for support and hospitality during his stay.

\section{APPENDIX A: TWO-PHOTON QUANTUM OPTICAL FORMALISM}

We use the two-photon formalism developed by Caves and Schumaker [9, 10 to describe GW interferometers with significant radiation-pressure effects. In this formalism, any quasi-monochromatic optical field $A$ with frequency near the carrier frequency $\omega$ is written as

$$
\begin{aligned}
E(t) & =E_{1}(t) \cos (\omega t)+E_{2}(t) \sin (\omega t) \\
& =\left[\begin{array}{ll}
\cos \omega t & \sin \omega t
\end{array}\right]\left[\begin{array}{l}
E_{1}(t) \\
E_{2}(t)
\end{array}\right],
\end{aligned}
$$

where $E_{1}(t)$ and $E_{2}(t)$ are called quadrature fields, which vary at timescales much longer than that of the optical oscillation, $1 / \omega$. The quadrature formalism replaces $E(t)$ by

$$
\mathbf{E}=\left[\begin{array}{c}
E_{1}(t) \\
E_{2}(t)
\end{array}\right] .
$$

The DC components of $E_{1,2}(t)$ can be regarded as monochromatic carrier light. In particular, carrier light with amplitude $D e^{i \varphi}$ is represented as

$$
D e^{i \varphi} \Leftrightarrow\left(D e^{i \varphi}\right) e^{-i \omega t} \Leftrightarrow D\left(\begin{array}{c}
\cos \varphi \\
\sin \varphi
\end{array}\right) .
$$

AC components of $E_{1,2}(t)$, which we denote by $A_{1,2}(t)$, are called sideband fields, which are usually more convenient to study once transformed into the frequency domain,

$$
\tilde{A}_{1,2}(\Omega)=\int_{-\infty}^{+\infty} A_{1,2}(t) e^{i \Omega t} d t
$$

In quantum two-photon optics, it is convenient to use a particular normalization for sideband fields:

$$
=\sqrt{\frac{4 \pi \hbar \omega}{\mathcal{A} c}} \int_{0}^{+\infty} \frac{d \Omega}{2 \pi}\left[a_{1,2}(\Omega) e^{-i \Omega t}+\text { H.c. }\right] .
$$


In this way, we have a convenient set of commutation relations (for $\Omega \ll \omega$ ) [9, 10]:

$$
\begin{gathered}
{\left[a_{1}, a_{1}^{\prime}\right]=\left[a_{2}, a_{2}^{\prime}\right]=\left[a_{1}, a_{1}^{\prime \dagger}\right]=\left[a_{2}, a_{2}^{\prime \dagger}\right]=0,} \\
{\left[a_{1}, a_{2}^{\prime \dagger}\right]=-\left[a_{2}, a_{1}^{\prime \dagger}\right]=2 \pi i \delta\left(\Omega-\Omega^{\prime}\right) .}
\end{gathered}
$$

[1] P. Fritschel, Proc. SPIE 4856-39, 282 (2002).

[2] B. Barish and R. Weiss, Physics Today 52, 44 (1999).

[3] V.B.Braginsky, F.Ya.Khalili, "Quantum Measurement," Cambridge University Press, (1992).

[4] J.H. Kimble, Y. Levin, A.B. Matsko, K.S. Thorne and S.P. Vyatchanin, Phys. Rev. D 65, 022002 (2002).

[5] A. Buonanno and Y. Chen, Phys. Rev. D 64, 042006 (2001).

[6] M. W. Regehr, "Signal extraction and control for an interferometric gravitational wave detector," Ph.D. thesis, California Institute of Technology, 1995; M. W. Regehr, J. E. Mason, and H. Yamamoto, "Twiddle: A program for analyzing interferometer frequency response," LIGOT990022-00-R.

[7] A. Freise, G. Heinzel, H. Lueck, R. Schilling, B. Willke, and K. Danzmann, Class. Quant. Grav. 21, 1067 (2004).

[8] B. Bhawal, M. Evans, M. Rakhmanov, V. Sannibale, and H. Yamamoto, "The LIGO end-to-end simulation program," Proc. Moriond workshop on gravitational-waves and experimental gravity (2003).

[9] C. M. Caves and B. L. Schumaker, Phys. Rev. A 31, 3068 (1985).

[10] B. S. Schumaker and C. M. Caves, Phys. Rev. A 31, 3093
Here we have denoted $a_{1,2} \equiv a_{1,2}(\Omega), a_{1,2}^{\prime} \equiv a_{1,2}\left(\Omega^{\prime}\right)$. (1985).

[11] J. Harms, R. Schnabel, K. Danzmann, Phys. Rev. D 70, 102001 (2004) .

[12] R.W.P. Drever, "Interferometric Detectors for Gravitational Ratiaion", in Gravitational Radiation, edited by N. Deruelle and T. Piran (North-Holland, Amsterdam, 1983), p 321-338.

[13] T. Corbitt, Y. Chen, D. Ottaway, S. Whitcomb, and N. Mavalvala, "A ponderomotively squeezed source for advanced gravitational-wave detectors," in preparation (2004).

[14] A. Buonanno and Y. Chen, Phys. Rev. D 67, 062002 (2003).

[15] V.B. Braginsky, F.Ya. Khalili, Phys. Lett. A 257, 241246 (1999).

[16] Strictly speaking, we have to consider the fourdimensional linear space spanned by $a(\omega+\Omega), a(\omega-\Omega)$ and their Hermitian conjugates, $a^{\dagger}(\omega+\Omega), a^{\dagger}(\omega-\Omega)$. However, the fact that the sideband fields are real functions in the time domain will limit us to a twodimensional subspace. 